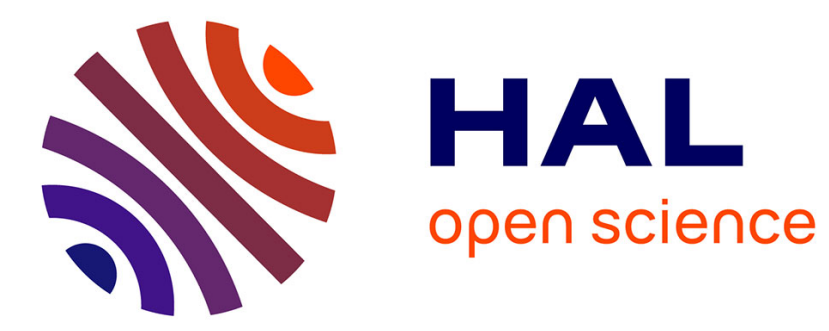

\title{
Homogenization of elasto-(visco) plastic composites based on an incremental variational principle
}

\author{
L. Brassart, Laurent Stainier, I. Doghri, L. Delannay
}

\section{To cite this version:}

L. Brassart, Laurent Stainier, I. Doghri, L. Delannay. Homogenization of elasto-(visco) plastic composites based on an incremental variational principle. International Journal of Plasticity, 2012, 36, pp.86-112. 10.1016/j.ijplas.2012.03.010 . hal-01006931

\section{HAL Id: hal-01006931 \\ https://hal.science/hal-01006931}

Submitted on 24 Jun 2019

HAL is a multi-disciplinary open access archive for the deposit and dissemination of scientific research documents, whether they are published or not. The documents may come from teaching and research institutions in France or abroad, or from public or private research centers.
L'archive ouverte pluridisciplinaire HAL, est destinée au dépôt et à la diffusion de documents scientifiques de niveau recherche, publiés ou non, émanant des établissements d'enseignement et de recherche français ou étrangers, des laboratoires publics ou privés. 


\title{
Homogenization of elasto-(visco) plastic composites based on an incremental variational principle
}

\author{
L. Brassart ${ }^{\text {a }}$, L. Stainier ${ }^{\text {b }}$, I. Doghri ${ }^{\text {a }}$, L. Delannay ${ }^{\text {a }}$ \\ a Université catholique de Louvain, iMMC, 4 Av. Georges Lemaître, B-1348 Louvain-la-Neuve, Belgium \\ ${ }^{\mathrm{b}}$ Ecole Centrale de Nantes, GeM (UMR 6183 CNRS), 1 Rue de la Noë, BP 92101, F-44321 Nantes, France
}

An original approach is proposed in order to compute the homogenized response of composite materials with elasto-(visco) plastic constituents. The formulation is based on an incremental variational principle according to which the local stress-strain relation derives from a single incremental potential constructed from a free energy and a dissipation function. Both rate-dependent and rate-independent plasticity are handled within the same framework through the choice of the dissipation function. The key feature of the model is the explicit use of the elastic trial strain in order to define a Linear Comparison Composite whose mechanical response coincides with the response of the actual composite at a given time step. The hereditary character of the behavior is accounted for through internal variables. The method was successfully applied to several two-phase elasto-plastic and elasto-viscoplastic composites made of a continuous matrix reinforced by ellipsoidal inclusions. General loading conditions, including cyclic ones, were considered. The proposed method provides accurate predictions of the macroscopic response in many cases, and competes with previously proposed schemes in elasto-(visco) plasticity.
Keywords:

Micromechanics

Plasticity

Viscoplasticity

Creep

Heterogeneous media

\section{Introduction}

Mean-Field (MF) homogenization methods are efficient micromechanical approaches enabling two-scale simulations of composite parts and structures at reasonable computational cost. Contrarily to full-field simulations on a Representative Volume Element (RVE) of the microstructure, they do not provide a complete description of the local fields, but focus on field statistics in the phases, typically on first and second moments of strain and stress fields (that is, their mean and variance). Although reliable MF models for linear elastic composites have been available for a long time, developing MF approaches for nonlinear composites remains highly challenging. In this case, intra-phase field fluctuations have a major impact on the effective behavior. In addition, the problem complexity increases when the phase behavior is hereditary, as proper account of the loading history must then be supplied. This paper focuses on the MF homogenization of composites having elastoplastic and/or elasto-(visco) plastic constituents.

The proposed approach relies on the definition of linear comparison properties for each phase of the composite at a given stage of deformation. The comparison properties in turn define a so-called "Linear Comparison Composite" (LCC) whose mechanical response is (hopefully) close to the actual response of the nonlinear composite at the given load step. The effective behavior of the LCC can then be computed based on any linear MF model suited for the considered microstructure, such as the self-consistent estimate (Kröner, 1958; Hill, 1965b) or the Mori-Tanaka scheme (Mori and Tanaka, 1973). 
The most popular approaches of this kind rely on a direct linearization of the local stress-strain relation using tangent (Hill, 1965a; Molinari et al., 1987; Lebensohn and Tomé, 1993) or secant (Berveiller and Zaoui, 1979; Tandon and Weng, 1988; Suquet, 1995) operators. However, few of them can handle elasto-(visco) plasticity under general loading conditions. Hill's incremental formulation (Hill, 1965a) is well suited for rate-independent elasto-plasticity, as the constitutive equation can be written in rate form using the instantaneous (anisotropic) tangent operator. However, it is well-known that Hill's method yields overly stiff predictions (Gilormini, 1995; Suquet, 1996, 1997), unless some isotropic projection of the tangent operator is considered, instead of the exact, anisotropic expression (González and LLorca, 2000; Doghri and Ouaar, 2003; Pierard and Doghri, 2006b). Using this heuristic prescription, satisfying predictions have been obtained in numerous examples of two-phase composites (Doghri and Friebel, 2005; Pierard et al., 2007a). The formulation was recently enriched with second-order moments, which improves the predictions in case of highly contrasted phase properties (Doghri et al., 2011). Yet, the approach is not fully satisfying from a theoretical viewpoint, even if some justification for the isotropization has been proposed (Chaboche and Kanouté, 2003; Chaboche et al., 2005). The affine formulation of Masson et al. (2000) is an interesting alternative to Hill's formulation (see also (Zaoui, 2002)). It uses the (anisotropic) tangent operators but introduces polarization stresses. The method was modified to incorporate second-order moments by Brenner et al. (2001). However, when applied to elasto-plasticity, isotropized operators are still needed to avoid overly stiff predictions, as discussed in (Chaboche et al., 2005 and Rekik et al., 2007).

Hill's incremental method cannot directly be applied to rate-dependent elasto-plasticity, as no instantaneous tangent operator is available. Alternatively, secant (Li and Weng, 1997, 1998) and affine (Masson and Zaoui, 1999; Pierard and Doghri, 2006a; Pierard et al., 2007b) linearizations have been proposed to obtain a thermo-viscoelastic comparison material which can be homogenized resorting to the correspondence principle and the Laplace transform (Hashin, 1970). The main drawback of this approach is that it requires numerical inversion of the Laplace transform, which can be very costly. Recently, Doghri et al. (2010) proposed an "incrementally affine" procedure which involves the homogenization of a thermoelastic composite directly in the time-domain, thus avoiding the recourse to the Laplace transform. The method relies on an affine linearization of the constitutive equations directly in a time-discretized setting, exploiting the algorithmic structure of the constitutive equations. However, the method still requires the isotropization of the algorithmic tangent operator to obtain satisfying predictions.

Both rate-dependent and rate-independent elasto-plasticity can be handled within the framework of the Transformation Field Analysis (TFA) (Dvorak and Benveniste, 1992; Dvorak, 1992). In this framework, the plastic strain is considered as a uniform eigenstrain in each phase, so that the constitutive equations become thermoelastic. However, the TFA yields too stiff predictions (Suquet, 1997; Chaboche et al., 2001), which can be attributed to the assumption of uniform plastic strain in each phase. Recently, non-uniform TFA frameworks were developed for composites and polycrystals, enabling a better description of the plastic strain fields and giving reasonable predictions (Michel and Suquet, 2004; Franciosi and Berbenni, 2008; Fritzen and Böhlke, 2010). However, the method requires a preliminary full-field analysis in order to determine plastic modes.

A promising direction towards the development of advanced and rigorous MF models for hereditary behavior is provided by incremental variational principles. Such variational principles aim at recasting the constitutive equations into equivalent minimization problems (Comi et al., 1991; Ortiz and Stainier, 1999; Carstensen et al., 2002; Miehe, 2002). Therefore, they enable the generalization of the variational framework for the homogenization of nonlinear elastic composites (see e.g. the review of Ponte Castañeda and Suquet (1998)) to the inelastic case. Regarding the formulation of MF models, the variational framework is very attractive for several reasons. It is mathematically elegant, and enables the derivation of rigorous bounds on the effective behavior. It can also lead to the derivation of more sophisticated estimates which are not available from a direct linearization of the constitutive behavior. In particular, variational techniques for the MF homogenization of nonlinear elastic composites have been successfully developed (Ponte Castañeda, 1991, 1996, 2002). In principle, they can be extended to the inelastic case adopting the time-discretized setting, provided that a suitable treatment of the internal variable is supplied. For instance, incremental variational estimates were recently proposed by Lahellec and Suquet $(2007 a, b)$ in the context of nonlinear viscoelasticity.

The general objective of the present work is to propose new MF models for elasto-viscoplastic composites relying on an incremental variational principle. Here, we generalize our previous formulation for rate-independent elasto-plasticity (Brassart et al., 2011) in order to account for both rate-independent and rate-dependent elasto-plasticity within a common framework. The proposed method exploits the algorithmic elegance of the time-integration of $\mathrm{J}_{2}$ elasto-plasticity in order to define the linear comparison properties. The latter are characterized by the secant operators for the trial strain-stress relation evaluated at the second moments of the trial strain in the phase. The method also yields homogenized radial return equations for each phase, as well as a homogenized plastic flow rule. The method is verified by comparing its predictions to reference results obtained from FE simulations on multiparticle cells. Accurate predictions are obtained in most cases tested so far. In particular, the new method competes with incremental tangent approaches.

The paper is organized as follows. The incremental variational principle is described in Section 2 in the context of elasto-viscoplasticity at small strain. We show that this principle leads to radial return equations for the internal variables. The linearization method sustaining the homogenization model of Brassart et al. (2011) is briefly recalled in Section 3. The homogenization scheme is developed in Section 4, and its numerical implementation is discussed in Section 5 . The accuracy of the method is evaluated in Section 6 in several examples of particle-reinforced composites.

Throughout the paper, Einstein's convention is used, with indices ranging from 1 to 3 , unless otherwise indicated. The products of tensors are expressed as $(\boldsymbol{A}: \boldsymbol{\sigma})_{i j}=A_{i j k l} \sigma_{l k},(\boldsymbol{\sigma}: \boldsymbol{\sigma})=\sigma_{i j} \sigma_{j i}$, and $(\boldsymbol{\sigma} \otimes \boldsymbol{\sigma})_{i j k l}=\sigma_{i j} \sigma_{k l}$. The symbols 1 and $\boldsymbol{I}$ stand for 
the second and symmetric fourth order identity tensors, respectively. The spherical and deviatoric operators $\boldsymbol{I}^{\mathrm{vol}}$ and $\boldsymbol{I}^{\mathrm{dev}}$ are given by

$$
\boldsymbol{I}^{\mathrm{vol}} \equiv \frac{1}{3} \mathbf{1} \otimes \mathbf{1}, \quad \boldsymbol{I}^{\mathrm{dev}} \equiv \boldsymbol{I}-\boldsymbol{I}^{\mathrm{vol}} .
$$

The von Mises measures of stress and strain are respectively given by

$$
\sigma_{e q}=\left(\frac{3}{2} \boldsymbol{s}: \boldsymbol{s}\right)^{1 / 2} \text { and } \varepsilon_{e q}=\left(\frac{2}{3} \boldsymbol{e}: \boldsymbol{e}\right)^{1 / 2},
$$

where $\boldsymbol{s}$ and $\boldsymbol{e}$ denote the deviatoric parts of $\boldsymbol{\sigma}$ and $\boldsymbol{\varepsilon}$ :

$$
\boldsymbol{s}=\boldsymbol{I}^{\mathrm{dev}}: \boldsymbol{\sigma}, \quad \boldsymbol{e}=\boldsymbol{I}^{\mathrm{dev}}: \boldsymbol{\varepsilon} .
$$

\section{Variational formulation of the constitutive equations}

We begin by summarizing the variational formulation of the elasto-(visco) plastic equations. The formulation relies on the classical thermodynamic framework of Generalized Standard Media (Halphen and Nguyen, 1975; Germain et al., 1983), according to which the behavior is described by two potentials: a free energy and a dissipation function (see also the textbooks by Lemaître and Chaboche (1990) and Maugin (1992)). Within this framework, the incremental variational principle of Ortiz and Stainier (1999) yields constitutive updates for the stress and the internal variables. It suggests an algorithmic approach very similar in nature to the radial return scheme of Wilkins et al. (1964). These features are essential for the formulation of the homogenization method presented in the sequel.

\subsection{Continuum variational principle}

Under the small displacements hypothesis, the total strain tensor is first decomposed into elastic and (visco) plastic (inelastic) parts: $\varepsilon=\varepsilon^{e}+\varepsilon^{p}$. We assume the local state to be fully described by the total strain, the plastic strain, and a scalar variable $p$ measuring the accumulation of plastic deformation. The free energy function is split into elastic and plastic parts:

$$
\psi\left(\boldsymbol{\varepsilon}, \boldsymbol{\varepsilon}^{p}, p\right)=\underbrace{\frac{1}{2}\left(\boldsymbol{\varepsilon}-\boldsymbol{\varepsilon}^{p}\right): \boldsymbol{C}:\left(\boldsymbol{\varepsilon}-\boldsymbol{\varepsilon}^{p}\right)}_{\psi^{p}\left(\boldsymbol{\varepsilon}^{e}\right)}+\underbrace{\int_{0}^{p} R(q) d q}_{\psi^{p}(p)} .
$$

The latter expression accounts for isotropic hardening only. Kinematic hardening can be considered within the present framework by introducing a dependance of $\psi^{p}$ in $\varepsilon^{p}$ (for linear kinematic hardening of the Prager type) or in an additional strain-like internal variable (for nonlinear kinematic hardening) ${ }^{1}$ (see for instance Lemaître and Chaboche (1990)). However, kinematic hardening will not be considered in the present work. The von Mises plasticity model can be characterized by a flow rule of the form:

$$
\dot{\boldsymbol{\varepsilon}}^{p}=\dot{p} \boldsymbol{N} \quad \text { with } \quad \operatorname{tr} \boldsymbol{N}=0 \text { and } \boldsymbol{N}: \boldsymbol{N}=\frac{3}{2},
$$

where $\boldsymbol{N}$ is a kinematic variable. The first constraint on $\boldsymbol{N}$ ensures incompressibility of plastic flow and the second one ensures the uniqueness of the parametrization (5). It follows that: $\dot{p}=\left((2 / 3) \dot{\boldsymbol{\varepsilon}}^{p}: \dot{\boldsymbol{\varepsilon}}^{p}\right)^{1 / 2}$, so that the scalar variable $p$ is the classical accumulated plastic strain. Thus, in the present framework, the internal variables to be determined through the variational principle are the direction of plastic flow $\boldsymbol{N}$ and the rate of accumulated plastic strain $\dot{p}$, the rate of plastic strain tensor being given by the flow rule (5). State laws and evolution laws must ensure that the mechanical dissipation is always positive:

$$
\mathcal{D}=\boldsymbol{\sigma}: \dot{\boldsymbol{\varepsilon}}^{p}-R \dot{p}=Y(\boldsymbol{N}) \dot{p} \geqslant 0,
$$

where $Y(\boldsymbol{N}) \equiv \boldsymbol{\sigma}: \boldsymbol{N}-R$.

Following Ortiz and Stainier (1999), a stress power density function is introduced:

$$
J(\dot{\boldsymbol{\varepsilon}}, \dot{p}, \boldsymbol{N})=\dot{\psi}\left(\dot{\boldsymbol{\varepsilon}}, \dot{\boldsymbol{\varepsilon}}^{p}(\dot{p}, \boldsymbol{N}), \dot{p}\right)+\phi^{*}(\dot{p})=\boldsymbol{\sigma}: \dot{\boldsymbol{\varepsilon}}-Y(\boldsymbol{N}) \dot{p}+\phi^{*}(\dot{p}),
$$

where $\phi^{*}(\dot{p})$ is a dissipation function. Note that in general, the dissipation function may also depend on the state variables as parameters. The dissipation function will be specified hereafter. For a given strain rate $\dot{\boldsymbol{\varepsilon}}$, evolution laws are found from the following variational principle:

$$
\inf _{\dot{p}, \boldsymbol{N}} J(\dot{\boldsymbol{\varepsilon}}, \dot{p}, \mathbf{N}) \equiv W(\dot{\boldsymbol{\varepsilon}})
$$

\footnotetext{
${ }^{1}$ Incremental variational principles accounting for nonlinear kinematic hardening have been proposed only recently (Mosler, 2010; Canadija and Mosler, 2011).
} 
where the minimization is performed under constraints (5). The optimality condition with respect to (w.r.t.) $\dot{p}$ yields the following kinetic relations:

$$
Y=\frac{\partial \phi^{*}}{\partial \dot{p}}(\dot{p}), \quad \text { or, equivalently, } \quad \dot{p}=\frac{\partial \phi}{\partial Y}(Y),
$$

where $\phi$ is the dual of $\phi^{*}$ by the Legendre transform. By choosing $\phi^{*}(\dot{p})$ to be non-negative, convex and such that $\phi^{*}(0)=0$, the mechanical dissipation (6) is necessarily non-negative. The minimization of $J$ w.r.t. $\boldsymbol{N}$ under constraints (5) is performed using Lagrange multipliers and it yields (for computation details, see Brassart (2011), Appendix C):

$$
\boldsymbol{N}=\frac{3}{2} \frac{\boldsymbol{s}}{\sigma_{e q}}
$$

The minimization of $J$ w.r.t. $N$ can be interpreted as a maximum dissipation principle at fixed $\dot{p}$. Finally, by taking stationarity w.r.t. $\dot{p}$ and $\boldsymbol{N}$ into account, it immediately appears from (7) that $W$ acts as a potential for the stress:

$$
\boldsymbol{\sigma}=\frac{d W}{d \dot{\boldsymbol{\varepsilon}}}(\dot{\boldsymbol{\varepsilon}})
$$

\subsection{Dissipation function in rate-dependent and rate-independent elasto-plasticity}

Retrieving the classical equations of elasto-viscoplasticity requires us to specify the form of the dissipation function. A sufficiently general form for the present work includes a parametric dependance on $p$ :

$$
\phi^{*}(\dot{p} ; p)= \begin{cases}h_{v}(\dot{p} ; p) & \dot{p} \geqslant 0, \\ +\infty & \text { otherwise. }\end{cases}
$$

Here, the viscous potential $h_{v}(\dot{p} ; p)$ is non-negative, convex w.r.t. $\dot{p}$ and chosen such that:

$$
\left.\frac{\partial h_{v}}{\partial \dot{p}}\right|_{\dot{p}=0^{+}}=\sigma_{Y},
$$

where $\sigma_{Y}$ is the initial yield stress. The dissipation function and its dual are represented in Fig. 1(a) and (b). Since $\phi^{*}$ is not differentiable at $\dot{p}=0$, the partial derivative in (9) must be understood in the sense of sub-differential. The following properties follow from the specific form of $\phi^{*}$ :

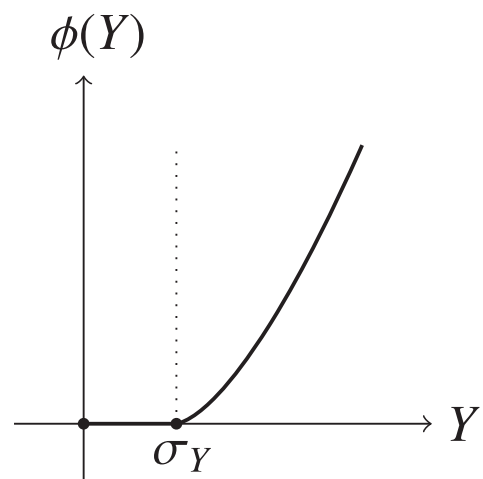

(a)

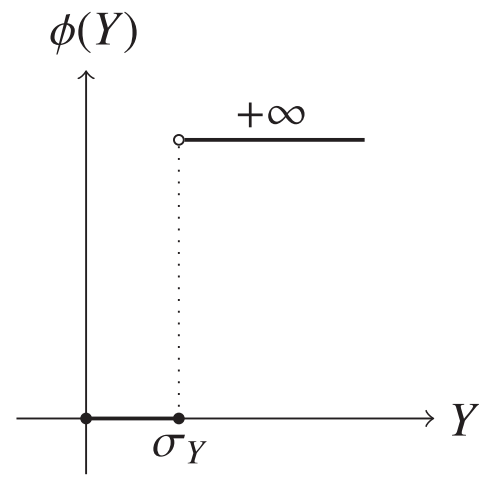

(c)

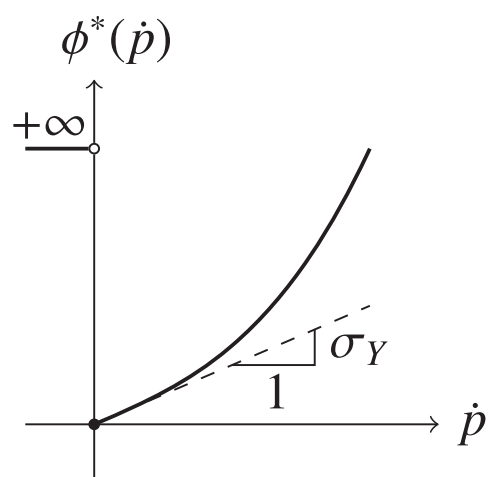

(b)

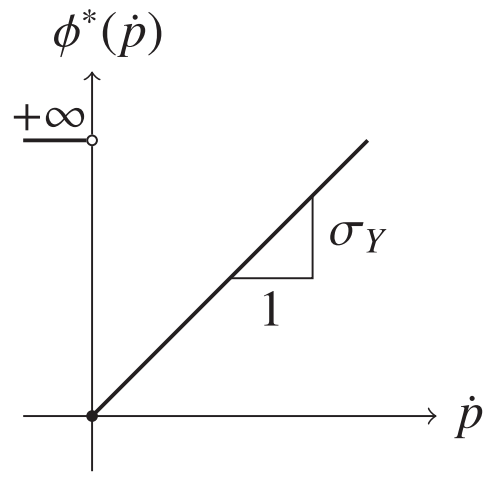

(d)

Fig. 1. Dual dissipation functions for rate-dependent ((a) and (b)) and rate-independent ((c) and (d)) elasto-plasticity. 


$$
\begin{aligned}
& Y=\sigma_{e q}-R<\sigma_{Y} \Longleftrightarrow \dot{p}=0, \\
& Y=\sigma_{e q}-R \geqslant \sigma_{Y} \Longleftrightarrow \dot{p}>0 .
\end{aligned}
$$

In other words, deformation is purely elastic when the force is inside the elasticity domain $\left[0, \sigma_{Y}\right]$. Plastic flow occurs as soon as the force reaches the yield stress $\sigma_{Y}$. Negative values of $\dot{p}$ are prohibited, as they would imply infinite dissipation. Rateindependent plasticity is obtained as a particular case of viscoplasticity by choosing $\phi^{*}$ homogeneous of degree one w.r.t. $\dot{p}$ :

$$
\phi^{*}(\dot{p})= \begin{cases}\sigma_{Y} \dot{p} & \dot{p} \geqslant 0 \\ +\infty & \text { otherwise }\end{cases}
$$

In this case, the force $Y$ may not leave the yield surface during plastic flow. The dissipation functions for rate-independent plasticity are depicted in Fig. 1(c) and (d). Note that in the case of rate-independent elasto-plasticity, the functional $J$ (7) is not convex w.r.t. $\dot{p}$, so that the minimization w.r.t. $\dot{p}$ cannot be enforced in general (to the contrary of the minimization w.r.t. $\boldsymbol{N})$. Therefore, only stationarity in this variable is imposed in the variational principle (8).

\subsection{Discretized setting}

The continuous variational principle (8) serves as the basis for the derivation of constitutive updates in a time-discretized setting. The state at time $t_{n}$ is supposed to be given: $\left\{\boldsymbol{\varepsilon}_{n}, \boldsymbol{\varepsilon}_{n}^{p}, p_{n}\right\}$, as well as the total strain at $t_{n+1}: \boldsymbol{\varepsilon}_{n+1}$. In order to compute the stress $\boldsymbol{\sigma}_{n+1}$ and internal variables $\boldsymbol{\varepsilon}_{n+1}^{p}$ and $p_{n+1}$, we assume that $\dot{p}$ is constant over the time step and given by the ratio $\Delta p / \Delta t$, with $\Delta()=()_{n+1}-()_{n}$. Similarly, the plastic flow rule (5) is discretized as:

$$
\Delta \boldsymbol{\varepsilon}^{p}=\Delta p \boldsymbol{N},
$$

where $\mathbf{N}$ now denotes an (a priori unknown) constant direction of plastic flow for the time increment subjected to the same constraints as in the continuous case. The incremental variational principle of Ortiz and Stainier (1999) reads:

$$
W_{\Delta}\left(\boldsymbol{\varepsilon}_{n+1}\right)=\inf _{\Delta p, \boldsymbol{N}} J_{\Delta}\left(\boldsymbol{\varepsilon}_{n+1}, \Delta p, \boldsymbol{N}\right)
$$

where the functional $J_{\Delta}$ is obtained by integrating expression (7) over the time step:

$$
J_{\Delta}\left(\boldsymbol{\varepsilon}_{n+1}, \Delta p, \boldsymbol{N}\right)=\psi\left(\boldsymbol{\varepsilon}_{n+1}, \boldsymbol{\varepsilon}_{n+1}^{p}(\Delta p, \boldsymbol{N}), p_{n+1}\right)-\psi_{n}+\Delta t \phi^{*}\left(\frac{\Delta p}{\Delta t} ; p_{n+\theta}\right) .
$$

Here $\psi_{n}$ is the free energy computed for the state variables at $t_{n}$. The possible dependence of $\phi^{*}$ in $p$ is handled by evaluating the dissipation function at an intermediary value during the time interval (generalized midpoint rule):

$$
p_{n+\theta}=(1-\theta) p_{n}+\theta p_{n+1} \text {. }
$$

Stationarity of $J_{\Delta}$ w.r.t. $\Delta p$ leads to:

$$
Y_{n+1}=\frac{\partial \phi^{*}}{\partial \dot{p}}\left(\frac{\Delta p}{\Delta t} ; p_{n+\theta}\right)+\theta \Delta t \frac{\partial \phi^{*}}{\partial p}\left(\frac{\Delta p}{\Delta t} ; p_{n+\theta}\right)
$$

where $Y_{n+1}$ is defined similarly as in the continuous case: $Y_{n+1}=\boldsymbol{\sigma}_{n+1}: \boldsymbol{N}-R\left(p_{n+1}\right)$. The minimization w.r.t. $\boldsymbol{N}$ under constraints (5) is performed using Lagrange multipliers, and it yields (for computation details, see Brassart et al. (2011), Appendix C):

$$
\boldsymbol{N}=\frac{3}{2} \frac{\boldsymbol{s}_{n+1}^{t r}}{\sigma_{e q, n+1}^{t r}}=\frac{\boldsymbol{e}_{n+1}^{t r}}{\varepsilon_{e q, n+1}^{t r}}
$$

where $\boldsymbol{\sigma}^{t r}$ and $\boldsymbol{\varepsilon}^{\text {tr }}$ are the trial (or predictor) stress and strain, respectively:

$$
\boldsymbol{\sigma}_{n+1}^{t r}=\boldsymbol{C}: \boldsymbol{\varepsilon}_{n+1}^{t r}, \quad \boldsymbol{\varepsilon}_{n+1}^{t r}=\boldsymbol{\varepsilon}_{n+1}-\boldsymbol{\varepsilon}_{n}^{p}
$$

Note that (21) holds under the assumption of isotropic elasticity, in which case the elastic stiffness tensor expresses as: $\boldsymbol{C}=3 \kappa \boldsymbol{I}^{\mathrm{vol}}+2 \mu \boldsymbol{I}^{\mathrm{dev}}$, where $\kappa$ and $\mu$ are the bulk and shear moduli, respectively. Substituting expression (21) for $\boldsymbol{N}$ into the kinetic relation (20), the stationarity condition for $\Delta p$ becomes:

$$
-\sigma_{e q, n+1}^{t r}+3 \mu \Delta p+R\left(p_{n+1}\right)+\frac{\partial \phi^{*}}{\partial \dot{p}}\left(\frac{\Delta p}{\Delta t} ; p_{n+\theta}\right)+\underbrace{\theta \Delta t \frac{\partial \phi^{*}}{\partial p}\left(\frac{\Delta p}{\Delta t} ; p_{n+\theta}\right)}_{\mathcal{O}(\Delta t)}=0,
$$

where $\Delta p$ is necessarily non-negative by virtue of (12). The problem of the non-smoothness of the dissipation function for $\Delta p=0$ can be circumvented by evaluating the slope of the functional $J_{\Delta}$ for $\Delta p=0^{+}$. If it is negative, i.e. if

$$
-\sigma_{e q, n+1}^{t r}+R\left(p_{n}\right)+\frac{\partial \phi^{*}}{\partial \dot{p}}\left(0^{+} ; p_{n}\right)<0
$$


then the optimal $\Delta p$ is positive and satisfies condition (23). Otherwise, the optimal $\Delta p$ is zero and the increment is fully elastic. Finally, taking the stationarity of $W_{\Delta}$ w.r.t. $\Delta p$ and $\boldsymbol{N}$ into account, it is readily seen that the function $W_{\Delta}$ acts as an (incremental) potential for the stress:

$$
\boldsymbol{\sigma}_{n+1}=\frac{d W_{\Delta}}{d \boldsymbol{\varepsilon}_{n+1}}\left(\boldsymbol{\varepsilon}_{n+1}\right)=\frac{\partial J_{\Delta}}{\partial \boldsymbol{\varepsilon}_{n+1}}\left(\boldsymbol{\varepsilon}_{n+1}, \Delta p, \boldsymbol{N}\right)=\boldsymbol{C}:\left(\boldsymbol{\varepsilon}_{n+1}-\boldsymbol{\varepsilon}_{n+1}^{p}\right),
$$

where $\Delta p$ and $\boldsymbol{N}$ are the solutions of the minimization problem (17).

The numerical scheme for the update of $\Delta p$ can be compared to the classical radial return equations (Wilkins et al., 1964, see also Simo and Hughes, 1998; Doghri, 2000). In the rate-independent case, the dissipation function (15) has no parametric dependence in $p$, and Eq. (23) simply reduces to:

$$
-\sigma_{e q, n+1}^{t r}+3 \mu \Delta p+R\left(p_{n+1}\right)+\underbrace{\frac{\partial \phi^{*}}{\partial \dot{p}}\left(\frac{\Delta p}{\Delta t}\right)}_{=\sigma_{Y} \text { if } \Delta p>0}=0,
$$

which coincides exactly with the classical radial return condition. In the case of rate-dependent plasticity, the dissipation function may include a parametric dependence in $p$, according to expression (12). The update of accumulated plastic strain then obeys the discretized kinetic Eq. (20). Clearly, this discretized flow rule does not coincide with its continuous expression computed for the time $t_{n+1}$ :

$$
Y_{n+1}=\frac{\partial \phi^{*}}{\partial \dot{p}}\left(\frac{\Delta p}{\Delta t}, p_{n+1}\right)
$$

which is fully implicit, contrarily to expression (20) (at least, for arbitrary $\theta$ ). In the case of the Perzyna-type viscoplastic function with a drag stress considered in Section 6.1, Brassart and Stainier (2012) suggested an optimal value of the integration parameter in terms of the viscoplastic exponent $M \in[0,1]$ :

$$
\theta^{*}=\frac{M+1}{M+2} \in[1 / 2,2 / 3] .
$$

This value of the integration parameter cancels the integration error in the case of linear hardening. In the sequel, integration of the incremental variational principle makes use of this value. The integration error is in $\mathcal{O}(\Delta t)$, so that is becomes negligible for reasonably small time steps, and the method remains consistent for any value of $\theta$. The impact of the integration parameter on the convergence of the variational updates is discussed in Brassart and Stainier (2012).

\section{Secant linearization based on the trial strain}

\subsection{Variational procedure}

The incremental homogenization procedure relies on the notion of linear comparison material, the properties of which need to be defined at each position and time. In the present section, the incremental variational principle (17) is reformulated in terms of the potential of the linear comparison material. To this end, we focus on the elastic part of the free energy $\psi^{e}$ which dictates the stress-strain relation for a fixed plastic strain $\boldsymbol{\varepsilon}_{n+1}^{p}$. Unless otherwise indicated, all quantities are evaluated at $t_{n+1}$ and the subscript is omitted for conciseness.

As detailed in Brassart et al. (2011), using expression (21) for $\mathbf{N}$, the elastic energy may be reexpressed in terms of the trial strain $\boldsymbol{\varepsilon}^{\text {tr }}$ and $\Delta p$ as:

$$
\tilde{\psi}^{e}\left(\boldsymbol{\varepsilon}^{t r}, \Delta p\right)=\frac{9}{2} \kappa\left(\varepsilon_{m}^{t r}\right)^{2}+f\left(\left(\varepsilon_{e q}^{t r}\right)^{2}, \Delta p\right)
$$

where the mean strain is defined as: $\varepsilon_{m} \equiv \operatorname{tr}(\varepsilon) / 3$ and the function $f$ is given by

$$
f\left(\left(\varepsilon_{e q}^{t r}\right)^{2}, \Delta p\right)=\frac{3}{2} \mu\left(1-\frac{\Delta p}{\sqrt{\left(\varepsilon_{e q}^{t r}\right)^{2}}}\right)^{2}\left(\varepsilon_{e q}^{t r}\right)^{2}=\frac{3}{2} \mu\left(\sqrt{\left(\varepsilon_{e q}^{t r}\right)^{2}}-\Delta p\right)^{2}
$$

The function $f$ is non-negative and convex w.r.t. $\left(\varepsilon_{e q}^{t r}\right)^{2}$. Considering the trial strain, instead of the total strain, as primary variable, expression (29) is similar in form to the elastic energy of a nonlinear, isotropic elastic material. The response is linear for purely hydrostatic loading and it is nonlinear in shear.

The technique used in order to define a linear comparison material inspires from the variational technique of Ponte Castañeda (1991) and is based on the following equality:

$$
f\left(\left(\varepsilon_{e q}^{t r}\right)^{2}, \Delta p\right)=f^{* *}\left(\left(\varepsilon_{e q}^{t r}\right)^{2}, \Delta p\right)=\sup _{\mu_{0}}\left\{\frac{3}{2} \mu_{0}\left(\varepsilon_{e q}^{t r}\right)^{2}-f^{*}\left(\frac{3}{2} \mu_{0}, \Delta p\right)\right\}
$$


Here $f^{*}$ is the convex dual of $f$ by Legendre transform (where $\Delta p$ acts as a parameter):

$$
f^{*}\left(\frac{3}{2} \mu_{0}, \Delta p\right)=\sup _{\left(\varepsilon_{e q}^{t r}\right)^{2}}\left\{\frac{3}{2} \mu_{0}\left(\varepsilon_{e q}^{t r}\right)^{2}-f\left(\left(\varepsilon_{e q}^{t r}\right)^{2}, \Delta p\right)\right\}=\frac{3}{2} \frac{\mu \mu_{0}(\Delta p)^{2}}{\left(\mu-\mu_{0}\right)} .
$$

Then, the elastic energy (29) may be reexpressed as:

$$
\tilde{\psi}^{e}\left(\boldsymbol{\varepsilon}^{t r}, \Delta p\right)=\sup _{\mu_{0}}\left\{W_{0}\left(\boldsymbol{\varepsilon}^{t r}, \mu_{0}\right)-f^{*}\left(\frac{3}{2} \mu_{0}, \Delta p\right)\right\},
$$

where $W_{0}$ is the energy of a fictitious, isotropic linear elastic material:

$$
W_{0}\left(\boldsymbol{\varepsilon}^{t r}, \mu_{0}\right)=\frac{1}{2} \boldsymbol{\varepsilon}^{t r}: \boldsymbol{C}_{0}: \boldsymbol{\varepsilon}^{t r} \quad \text { with } \quad \boldsymbol{C}_{0} \equiv 3 \kappa \boldsymbol{I}^{\mathrm{vol}}+2 \mu_{0} \boldsymbol{I}^{\mathrm{dev}},
$$

so that the new variable $\mu_{0}$ introduced by the Legendre transform is an effective shear modulus.

\subsection{Interpretation as a trial secant linearization}

The tensor $\boldsymbol{C}_{0}$ can be interpreted as a secant stiffness tensor relating the trial strain and the stress. Indeed:

$$
\boldsymbol{\sigma}=\frac{\partial \psi^{e}}{\partial \boldsymbol{\varepsilon}^{e}}=\frac{\partial \tilde{\psi}^{e}}{\partial \boldsymbol{\varepsilon}^{t r}}: \frac{\partial \boldsymbol{\varepsilon}^{t r}}{\partial \boldsymbol{\varepsilon}}=\boldsymbol{C}_{0}: \boldsymbol{\varepsilon}^{t r} .
$$

Contrarily to other secant schemes for nonlinear elasticity (Berveiller and Zaoui, 1979; Tandon and Weng, 1988; Suquet, 1995), the present secant method remains incremental in nature, as the trial strain depends on the plastic strain of the previous time step. The linearization method is illustrated in Fig. 2.

An analytical expression of the shear modulus can be derived from (33):

$$
\left.\mu_{0}=\mu \quad 1-\frac{\Delta p}{\varepsilon_{e q}^{t r}}\right) \text {. }
$$

This modulus does not coincide with the secant elasto-plastic shear modulus obtained in a deformation theory of plasticity, which writes:

$$
\mu^{\mathrm{sec}}=\mu\left(\frac{\sigma_{e q}}{\sigma_{e q}+3 \mu p}\right) .
$$

It differs also from the shear modulus $\mu^{\text {tgt }}$ of the algorithmic tangent operator of elasto-plasticity, when isotropized according to the spectral method (see Appendix C, Eq. (97)):

$$
\mu^{\mathrm{tgt}}=\mu\left(1-\frac{3 \mu}{3 \mu+R^{\prime}(p)}\right) .
$$

It turns out that the effective shear modulus (36) is identical to coefficient $k_{2}$ in decomposition (94) of the algorithmic tangent operator according to the spectral method.

\section{Homogenization}

\subsection{Incremental variational formulation of the homogenization problem}

We now consider a RVE of a composite microstructure with volume $V$. The spatial arrangement of the phases is described by characteristic functions $\chi^{(r)}(\boldsymbol{x})$ such that $\chi^{(r)}(\boldsymbol{x})=1$ if $\boldsymbol{x}$ is in phase $r$, and zero otherwise. The volume fraction of phase $r$ is

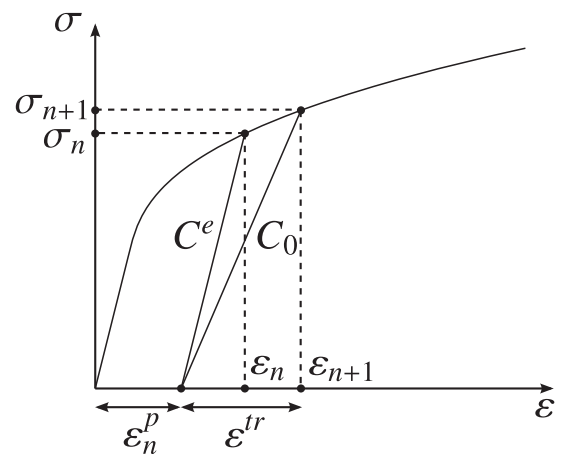

Fig. 2. The proposed variational formulation can be interpreted as a secant method based on the elastic trial strain: $\boldsymbol{\varepsilon}_{n+1}^{t r}=\boldsymbol{\varepsilon}_{n+1}-\boldsymbol{\varepsilon}_{n}^{p}$. 
$c_{r}=V^{(r)} / V$, where $V^{(r)}$ is the volume occupied by phase $r$. We make no particular assumption about the type of microstructure at this point. Volume averages over the RVE and phase $r$ of the composite are respectively denoted by $\langle\cdot\rangle$ and $\langle\cdot\rangle_{r}$. The free energy $\psi$ and dissipation function $\phi^{*}$ at some position $\boldsymbol{x}$ are written in the condensed form:

$$
\psi\left(\boldsymbol{x}, \boldsymbol{\varepsilon}, \boldsymbol{\varepsilon}^{p}, p\right)=\sum_{r=1}^{N} \chi^{(r)}(\boldsymbol{x}) \psi^{(r)}\left(\boldsymbol{\varepsilon}, \boldsymbol{\varepsilon}^{p}, p\right), \quad \phi^{*}(\boldsymbol{x}, \dot{p} ; p)=\sum_{r=1}^{N} \chi^{(r)}(\boldsymbol{x}) \phi^{*(r)}(\dot{p} ; p),
$$

where $\psi^{(r)}$ and $\phi^{*(r)}$ characterize the behavior of phase $r$.

The RVE is subjected to a given history of imposed macroscopic deformation $\overline{\boldsymbol{\varepsilon}}(t)$. Boundary conditions (B.C.) are applied to the RVE in such a way that: $\langle\boldsymbol{\varepsilon}(t)\rangle \equiv \overline{\boldsymbol{\varepsilon}}(t)$. For instance, linear displacement B.C. can be considered, with: $\boldsymbol{u}(t)=$ $\overline{\boldsymbol{\varepsilon}}(t) \cdot \boldsymbol{x}$ on $\partial V$, the boundary of the RVE. We aim to compute the corresponding macroscopic stress history $\overline{\boldsymbol{\sigma}}(t) \equiv\langle\boldsymbol{\sigma}(t)\rangle$. Adopting a time-discretized setting, the problem can be restated into that of determining the macroscopic stress $\overline{\boldsymbol{\sigma}}_{n+1}$ at $t_{n+1}$, for given macroscopic strain $\overline{\boldsymbol{\varepsilon}}_{n+1}$ and state variables at $t_{n}$. The local fields $\boldsymbol{\varepsilon}_{n+1}(\boldsymbol{x})$ and $\boldsymbol{\sigma}_{n+1}(\boldsymbol{x})$ must satisfy the following set of equations:

$$
\left\{\begin{array}{ll}
\boldsymbol{\nabla} \cdot \boldsymbol{\sigma}_{n+1}=\mathbf{0} & \text { in } V, \\
\boldsymbol{\varepsilon}_{n+1}=\frac{1}{2}\left((\nabla \boldsymbol{u})_{n+1}+(\nabla \boldsymbol{u})_{n+1}^{T}\right) & \text { in } V, \\
\boldsymbol{\sigma}_{n+1}=\frac{\partial W_{\Delta}}{\partial \boldsymbol{\varepsilon}_{n+1}}\left(\boldsymbol{x}, \boldsymbol{\varepsilon}_{n+1}\right) & \text { in } V, \\
\left\langle\boldsymbol{\varepsilon}_{n+1}\right\rangle=\overline{\boldsymbol{\varepsilon}}_{n+1} & + \text { B.C. on } \partial V
\end{array},\right.
$$

where $W_{\Delta}\left(\boldsymbol{x}, \boldsymbol{\varepsilon}_{n+1}\right)$ is the local incremental potential:

$$
W_{\Delta}\left(\boldsymbol{x}, \boldsymbol{\varepsilon}_{n+1}\right)=\inf _{\Delta p, \boldsymbol{N}} J_{\Delta}\left(\boldsymbol{x}, \boldsymbol{\varepsilon}_{n+1}, \Delta p, \boldsymbol{N}\right)
$$

with

$$
J_{\Delta}\left(\boldsymbol{x}, \boldsymbol{\varepsilon}_{n+1}, \Delta p, \boldsymbol{N}\right)=\psi\left(\boldsymbol{x}, \boldsymbol{\varepsilon}, \boldsymbol{\varepsilon}^{p}, p\right)-\psi_{n}(\boldsymbol{x})+\Delta t \phi^{*}(\boldsymbol{x}, \dot{p} ; p) .
$$

The local problem (40) can be restated into the following equivalent variational representation (Miehe, 2002; Lahellec and Suquet, 2007a):

$$
\bar{W}_{\Delta}\left(\overline{\boldsymbol{\varepsilon}}_{n+1}\right)=\inf _{\varepsilon_{n+1} \in \mathcal{K}\left(\overline{\boldsymbol{\varepsilon}}_{n+1}\right)}\left\langle W_{\Delta}\left(\boldsymbol{x}, \boldsymbol{\varepsilon}_{n+1}\right)\right\rangle=\inf _{\varepsilon_{n+1} \in \mathcal{K}\left(\overline{\boldsymbol{\varepsilon}}_{n+1}\right)}\left\langle\inf _{\Delta p, \boldsymbol{N}} J_{\Delta}\left(\boldsymbol{x}, \boldsymbol{\varepsilon}_{n+1}, \Delta p, \boldsymbol{N}\right)\right\rangle,
$$

where the set of kinematically admissible strain fields $\mathcal{K}\left(\overline{\boldsymbol{\varepsilon}}_{n+1}\right)$ in (43) is given by

$$
\mathcal{K}\left(\overline{\boldsymbol{\varepsilon}}_{n+1}\right)=\left\{\boldsymbol{\varepsilon}_{n+1} \mid \boldsymbol{\varepsilon}_{n+1}=\frac{1}{2}\left((\nabla \boldsymbol{u})_{n+1}+(\nabla \boldsymbol{u})_{n+1}^{T}\right),\left\langle\boldsymbol{\varepsilon}_{n+1}\right\rangle=\overline{\boldsymbol{\varepsilon}}_{n+1}\right\} .
$$

The new quantity $\bar{W}_{\Delta}$ is the effective incremental potential of the composite, from which the macroscopic stress can be derived:

$$
\overline{\boldsymbol{\sigma}}_{n+1}=\frac{\partial \bar{W}_{\Delta}}{\partial \overline{\boldsymbol{\varepsilon}}_{n+1}}\left(\overline{\boldsymbol{\varepsilon}}_{n+1}\right) .
$$

This expression is consistent with a classical thermodynamic formulation according to which the (macro) stress is the force conjugated to the (macro) strain. Moreover, the macroscopic stress defined by (45) coincides with the average of the stress fields over the RVE, as required (Lahellec and Suquet, 2007a). Hence, the problem of computing the overall response of the composite amounts to solving the variational problem (43) at each time step, which itself involves a local optimization problem (41) w.r.t. the internal variables at every position. Instead of a computationally-costly numerical full-field solution, an approximate mean-field approach is developed in the sequel while taking advantage of the linear comparison material introduced in Section 3.

\subsection{Definition of the linear comparison composite}

In this section we propose a variational procedure aiming at introducing the effective potential of a Linear Comparison Composite (LCC) into formulation (43) of the homogenization problem. All quantities are computed at $t_{n+1}$, unless otherwise indicated.

Using (33), the functional $J_{\Delta}(\boldsymbol{x}, \boldsymbol{\varepsilon}, \Delta p, \boldsymbol{N})(42)$ is first rewritten as:

$$
J_{\Delta}(\boldsymbol{x}, \boldsymbol{\varepsilon}, \Delta p, \boldsymbol{N})=\sup _{\mu_{0}}\left\{W_{0}\left(\boldsymbol{x}, \boldsymbol{\varepsilon}-\boldsymbol{\varepsilon}_{n}^{p}, \mu_{0}\right)-f^{*}\left(\boldsymbol{x}, \frac{3}{2} \mu_{0}, \Delta p\right)+\psi^{p}\left(\boldsymbol{x}, p_{n}+\Delta p\right)-\psi_{n}(\boldsymbol{x})+\Delta t \phi^{*}\left(\boldsymbol{x}, \frac{\Delta p}{\Delta t}, p_{n+\theta}\right)\right\},
$$

where $\boldsymbol{N}$ is the solution of the local minimum problem in (43). Then, the effective incremental potential of the composite can be expressed in terms of the potential of a LCC by introducing (46) into (43) and by permuting the order of optimization over $\boldsymbol{\varepsilon}(\boldsymbol{x}), \Delta p(\boldsymbol{x})$ and $\mu_{0}(\boldsymbol{x})$. It yields: 


$$
\bar{W}_{\Delta}(\overline{\boldsymbol{\varepsilon}})=\inf _{\Delta p} \sup _{\mu_{0}}\left\{\inf _{\boldsymbol{\varepsilon} \in \mathcal{K}(\overline{\boldsymbol{\varepsilon}})}\left\langle W_{0}\left(\boldsymbol{x}, \boldsymbol{\varepsilon}-\boldsymbol{\varepsilon}_{n}^{p}, \mu_{0}\right)\right\rangle+\left\langle-f^{*}\left(\boldsymbol{x}, \frac{3}{2} \mu_{0}, \Delta p\right)+\psi^{p}\left(\boldsymbol{x}, p_{n}+\Delta p\right)-\psi_{n}(\boldsymbol{x})+\Delta t \phi^{*}\left(\boldsymbol{x}, \frac{\Delta p}{\Delta t}, p_{n+\theta}\right)\right\rangle\right\} .
$$

Next, we restrict the field of solutions $\mu_{0}(\boldsymbol{x})$ to piecewise uniform fields. It follows that $\Delta p$ and $p$ too are necessarily piecewise uniform. Indeed the optimization problem over $\Delta p$ in (47) is local, and all terms containing $\Delta p$ are piecewise uniform during the first time step. The corresponding estimate of the effective potential may be written into a condensed form as:

$$
\bar{W}_{\Delta}(\overline{\boldsymbol{\varepsilon}}) \approx \widetilde{W}_{\Delta}(\overline{\boldsymbol{\varepsilon}})=\inf _{\Delta p^{(s)}} \sup _{\mu_{0}^{(s)}}\left\{\bar{W}_{0}\left(\overline{\boldsymbol{\varepsilon}}, \mu_{0}^{(s)}\right)+\sum_{r=1}^{N} c_{r} V^{(r)}\left(\Delta p^{(r)}, \mu_{0}^{(r)}\right)\right\}
$$

where $\bar{W}_{0}$ is the effective potential of a LCC characterized by shear moduli $\mu_{0}^{(r)}$ and subjected to an eigenstrain field $\boldsymbol{\varepsilon}_{n}^{p}(\boldsymbol{x})$ :

$$
\bar{W}_{0}\left(\overline{\boldsymbol{\varepsilon}}, \mu_{0}^{(s)}\right)=\inf _{\boldsymbol{\varepsilon} \in \mathcal{K}(\overline{\boldsymbol{\varepsilon}})}\left\langle\sum_{r=1}^{N} \chi^{(r)}(\boldsymbol{x}) \frac{1}{2}\left(\boldsymbol{\varepsilon}-\boldsymbol{\varepsilon}_{n}^{p}\right): \boldsymbol{C}_{0}^{(r)}:\left(\boldsymbol{\varepsilon}-\boldsymbol{\varepsilon}_{n}^{p}\right)\right\rangle
$$

with

$$
\boldsymbol{C}_{0}^{(r)}=3 \kappa^{(r)} \boldsymbol{I}^{\mathrm{vol}}+2 \mu_{0}^{(r)} \boldsymbol{I}^{\mathrm{dev}}
$$

and

$$
V^{(r)}\left(\Delta p^{(r)}, \mu_{0}^{(r)}\right)=-f^{*(r)}\left(\frac{3}{2} \mu_{0}^{(r)}, \Delta p^{(r)}\right)+\psi^{p(r)}\left(p^{(r)}\right)-\left\langle\psi_{n}\right\rangle_{r}+\Delta t \phi^{*(r)}\left(\frac{\Delta p^{(r)}}{\Delta t}, p_{n+\theta}^{(r)}\right)
$$

The optimization condition for $\mu_{0}^{(r)}$ in (48) amounts to:

$$
\frac{\partial \bar{W}_{0}}{\partial \mu_{0}^{(r)}}-c_{r} \frac{\partial f^{*(r)}}{\partial \mu_{0}^{(r)}}=0
$$

The first term on the left-hand side member can be rewritten in terms of the second moment of the trial strain as:

$$
\frac{\partial \bar{W}_{0}}{\partial \mu_{0}^{(r)}}=\frac{3}{2} c_{r}\left\langle\left(\varepsilon_{e q}^{t r}\right)^{2}\right\rangle_{r}
$$

The proof is given in Appendix A. The second term is readily computed analytically from expression (32) of $f^{*}$. Combining results (52) and (53), the optimal comparison shear modulus writes:

$$
\mu_{0}^{(r)}=\mu^{(r)}\left(1-\frac{\Delta p^{(r)}}{\sqrt{\left\langle\left(\varepsilon_{e q}^{t r}\right)^{2}\right\rangle_{r}}}\right) .
$$

Taking into account the stationarity condition w.r.t. $\mu_{0}^{(r)}$, the optimization over $\Delta p^{(r)}$ yields the homogenized radial return condition:

$$
-3 \mu^{(r)} \sqrt{\left\langle\left(\varepsilon_{e q}^{t r}\right)^{2}\right\rangle_{r}}+3 \mu^{(r)} \Delta p^{(r)}+R^{(r)}\left(p^{(r)}\right)+\frac{\partial \phi^{*(r)}}{\partial \dot{p}}\left(\frac{\Delta p^{(r)}}{\Delta t}, p_{n+\theta}^{(r)}\right)+\underbrace{\theta \Delta t \frac{\partial \phi^{*(r)}}{\partial p}\left(\frac{\Delta p^{(r)}}{\Delta t}, p_{n+\theta}^{(r)}\right)}_{\mathcal{O}(\Delta t)}=0 .
$$

A solution $\Delta p^{(r)}>0$ is found if the homogenized yield criterion is satisfied:

$$
-3 \mu^{(r)} \sqrt{\left\langle\left(\varepsilon_{e q}^{t r}\right)^{2}\right\rangle_{r}}+R^{(r)}\left(p_{n}^{(r)}\right)+\sigma_{Y}^{(r)}<0 .
$$

Otherwise, the optimal $\Delta p^{(r)}$ is zero, as negative values are precluded by virtue of the specific form of the dissipation function. In Eq. (56), the second moment $\sqrt{\left\langle\left(\varepsilon_{e q}^{t r}\right)^{2}\right\rangle_{r}}$ is computed on the LCC characterized by the elastic shear moduli $\mu^{(r)}$. Indeed, $\mu_{0}^{(r)} \rightarrow \mu^{(r)}$ as $\Delta p^{(r)} \rightarrow 0$.

The estimate $\widetilde{W}_{\Delta}(48)$ of the effective incremental potential requires the computation of the effective potential of a LCC with fluctuating eigenstrain field $\boldsymbol{\varepsilon}_{n}^{p}(\boldsymbol{x})$. However, semi-analytical homogenization schemes for thermoelastic composites are available (to our knowledge) only for piecewise uniform eigenstrain. Therefore, a strategy must be proposed to approximate the potential $\bar{W}_{0}$. 


\subsection{Approximation of compatible trial strain field}

In order to simplify the LCC problem (49), we propose to use the trial strain as control variable (instead of the total strain), and to approximate it by a compatible strain field, assuming that there exists some displacement field $\boldsymbol{u}^{\text {tr }}$ such that:

$$
\boldsymbol{\varepsilon}^{\text {tr }} \approx \frac{1}{2}\left(\left(\nabla u^{\text {tr }}\right)+\left(\nabla u^{\text {tr }}\right)^{T}\right)
$$

Also, we approximate the effective potential of the LCC by

$$
\bar{W}_{0}\left(\overline{\boldsymbol{\varepsilon}}, \mu^{(s)}\right) \approx \widetilde{W}_{0}\left(\overline{\boldsymbol{\varepsilon}}^{t r}, \mu^{(s)}\right)=\inf _{\varepsilon^{t r} \in \mathcal{K}\left(\overline{\boldsymbol{\varepsilon}}^{t r}\right)}\left\langle\sum_{r=1}^{N} \chi^{(r)}(\boldsymbol{x}) \frac{1}{2} \boldsymbol{\varepsilon}^{t r}: \boldsymbol{C}_{0}^{(r)}: \boldsymbol{\varepsilon}^{t r}\right\rangle
$$

where $\boldsymbol{\varepsilon}^{\text {tr }}$ belongs to the set of strain fields which are compatible with some macroscopic strain $\overline{\boldsymbol{\varepsilon}}^{\text {tr }}$ which remains to be defined. According to definition (44) of this set, the macroscopic strain field is such that $\overline{\boldsymbol{\varepsilon}}^{\text {tr }}=\left\langle\boldsymbol{\varepsilon}^{t r}\right\rangle=\langle\boldsymbol{\varepsilon}\rangle-\left\langle\boldsymbol{\varepsilon}_{n}^{p}\right\rangle$. In order to enforce the expected equality $\langle\boldsymbol{\varepsilon}\rangle=\overline{\boldsymbol{\varepsilon}}$, which does no longer follow from the formulation (58) of the LCC problem, we define the macroscopic trial strain as:

$$
\overline{\boldsymbol{\varepsilon}}^{t r} \equiv \overline{\boldsymbol{\varepsilon}}-\left\langle\boldsymbol{\varepsilon}_{n}^{p}\right\rangle \text {. }
$$

The estimate for the effective energy of the nonlinear composite then becomes:

$$
\widetilde{W}_{\Delta}(\overline{\boldsymbol{\varepsilon}})=\inf _{\Delta p^{(s)}} \sup _{\mu_{0}^{(s)}}\left\{\widetilde{W}_{0}\left(\overline{\boldsymbol{\varepsilon}}^{t r}, \mu_{0}^{(s)}\right)+\sum_{r=1}^{N} c_{r} V^{(r)}\left(\mu_{0}^{(r)}, \Delta p^{(r)}\right)\right\} .
$$

Assumption (57) amounts to neglect both inter- and intra-phase plastic incompatibilities. Despite the crudeness of this working hypothesis, it will be shown in the sequel that the overall behavior of the composite is properly predicted in many cases.

\subsection{Summary of the mean-field model}

In Sections 4.2 and 4.3 we derived the estimate (60) of the effective incremental potential of the composite. The MF model suggested by this expression goes as follows.

According to Eq. (45), the macroscopic stress of the composite is computed from the estimate of the effective incremental potential (60) as:

$$
\overline{\boldsymbol{\sigma}}=\frac{\partial \widetilde{W}_{\Delta}}{\partial \overline{\boldsymbol{\varepsilon}}}=\frac{\partial \widetilde{W}_{0}}{\partial \overline{\boldsymbol{\varepsilon}}^{t r}}=\overline{\boldsymbol{C}}_{0}: \overline{\boldsymbol{\varepsilon}}^{t r},
$$

where the second equality follows from the stationarity of $\widetilde{W}_{\Delta}$ w.r.t. $\mu_{0}^{(r)}$ and $\Delta p^{(r)}$. From the approximation of compatible trial strain presented in the previous section, the effective potential of the LCC simply reads as:

$$
\widetilde{W}_{0}\left(\overline{\boldsymbol{\varepsilon}}^{t r}, \mu_{0}^{(s)}\right)=\frac{1}{2} \overline{\boldsymbol{\varepsilon}}^{t r}: \overline{\boldsymbol{C}}_{0}: \overline{\boldsymbol{\varepsilon}}^{t r},
$$

where $\overline{\boldsymbol{C}}_{0}$ is the effective stiffness of the LCC. The latter can be obtained from any homogenization scheme for linear elastic composite suited for the microstructure under consideration. If the strain concentration tensors $\boldsymbol{A}^{(r)}$ are available, then:

$$
\overline{\boldsymbol{C}}_{0}=\sum_{r=1}^{N} c_{r} \boldsymbol{C}_{0}^{(r)}: \boldsymbol{A}^{(r)}
$$

As the macroscopic stress of the nonlinear composite (subjected to $\overline{\boldsymbol{\varepsilon}}$ ) and the macroscopic stress of the LCC (subjected to $\overline{\boldsymbol{\varepsilon}}^{\text {tr }}$ ) coincide, it seems natural to compute the phase averages of the stress in the nonlinear composite by corresponding averages in the LCC. In addition, we assume that per-phase averages of the trial strain field are computed on the LCC. Then, we may write:

$$
\left\langle\boldsymbol{\varepsilon}^{t r}\right\rangle_{r}=\boldsymbol{A}^{(r)}: \overline{\boldsymbol{\varepsilon}}^{t r}
$$

and

$$
\langle\boldsymbol{\sigma}\rangle_{r}=\boldsymbol{C}_{0}^{(r)}:\left\langle\boldsymbol{\varepsilon}^{t r}\right\rangle_{r}
$$

The second moment of the trial strain involved in relations (54)-(56) can be derived from the expression of the effective stiffness $\overline{\boldsymbol{C}}_{0}$ as (Bobeth and Diener, 1986; Kreher, 1990; Ponte Castañeda and Suquet, 1998):

$$
\left\langle\left(\varepsilon_{e q}^{t r}\right)^{2}\right\rangle_{r}=\frac{1}{3 c_{r}} \overline{\boldsymbol{\varepsilon}}^{t r}: \frac{\partial \overline{\boldsymbol{C}}_{0}}{\partial \mu_{0}^{(r)}}: \overline{\boldsymbol{\varepsilon}}^{t r} .
$$


The proposed estimate requires the computation of the average plastic strain at each time step, see Eq. (59). Following Brassart et al. (2011), the average plastic strain increment in phase $r$ is given by

$$
\left\langle\Delta \boldsymbol{\varepsilon}^{p}\right\rangle_{r}=\left\langle\Delta p \frac{\boldsymbol{e}^{t r}}{\varepsilon_{e q}^{t r}}\right\rangle_{r}=\frac{\Delta p^{(r)}}{\sqrt{\left\langle\left(\varepsilon_{e q}^{t r}\right)^{2}\right\rangle_{r}}}\left\langle\boldsymbol{e}^{t r}\right\rangle_{r} .
$$

\section{Numerical implementation}

The MF model proposed in the previous section involves the resolution of nonlinear equations at each time step. The following algorithm is proposed.

Given a macroscopic deformation $\overline{\boldsymbol{\varepsilon}}_{n+1}$ and the phase averages of the internal variables at $t_{n}: p_{n}^{(r)}$ and $\left\langle\boldsymbol{\varepsilon}_{n}^{p}\right\rangle_{r}$, we aim to update the macroscopic stress $\overline{\boldsymbol{\sigma}}_{n+1}$ and the internal variables $\left\langle\boldsymbol{\varepsilon}_{n+1}^{p}\right\rangle_{r}$ and $p_{n+1}^{(r)}$. The homogenization procedure involves the following steps.

- Compute the average plastic strain at $t_{n}:\left\langle\boldsymbol{\varepsilon}_{n}^{p}\right\rangle=\sum_{r=1}^{N} c_{r}\left\langle\boldsymbol{\varepsilon}_{n}^{p}\right\rangle_{r}$ and the macroscopic trial strain $(59): \overline{\boldsymbol{\varepsilon}}_{n+1}^{t r}=\overline{\boldsymbol{\varepsilon}}_{n+1}-\left\langle\boldsymbol{\varepsilon}_{n}^{p}\right\rangle$.

- Elastic predictor step: Set $\mu_{0}^{(r)}=\mu^{(r)}$.

1. Compute the effective stiffness $\overline{\boldsymbol{C}}_{0}$ from the tensor $\boldsymbol{C}_{0}^{(r)}$ built on $\mu^{(r)}$.

2. Compute second moments of the trial strain according to (66).

3. Decide whether plasticity develops in phase $r$ according to the homogenized yield criterion (56). If the increment is elastic in phase $r$, then $\mu_{0}^{(r)}=\mu^{(r)}$ is the solution and $\Delta p^{(r)}=0$. Otherwise, $\mu_{0}^{(r)}$ and $\Delta p^{(r)}$ must be determined iteratively.

- Plastic corrector step: Initialization: $\mu_{0}^{(r)}=\mu^{(r)}$. Iteration (i) (upper index (i) omitted for simplicity).

- Compute the effective stiffness $\overline{\boldsymbol{C}}_{0}$ from the tensor $\boldsymbol{C}_{0}^{(r)}$ built on $\mu_{0}^{(r)}$.

- For each phase $r$ which deforms plastically:

1. Compute second moments of the trial strain according to (66).

2. Compute $\Delta p^{(r)}$ from $\left\langle\varepsilon_{e q}^{t r}\right\rangle$ and $\mu_{0}^{(r)}$ according to Eq. (54).

3. Compute the scalar residual:

$$
F^{(r)}=-3 \mu^{(r)} \sqrt{\left\langle\left(\varepsilon_{e q}^{t r}\right)^{2}\right\rangle_{r}}+3 \mu^{(r)} \Delta p^{(r)}+R^{(r)}\left(p^{(r)}\right)+\frac{\partial \phi^{*(r)}}{\partial \dot{p}}\left(\frac{\Delta p^{(r)}}{\Delta t}, p_{n+\theta}^{(r)}\right)+\underbrace{\theta \Delta t \frac{\partial \phi^{*(r)}}{\partial p}\left(\frac{\Delta p^{(r)}}{\Delta t}, p_{n+\theta}^{(r)}\right)}_{\mathcal{O}(\Delta t)} .
$$

- if $\left|F^{(r)}\right|<$ TOL $\forall r$, then exit the loop.

- else: new iteration with new $\mu_{0}^{(r)}$.

\section{- After convergence}

1. Compute the increment of average plastic strain (67).

2. Update the internal variables:

$$
\begin{aligned}
& \left\langle\boldsymbol{\varepsilon}^{p}\right\rangle_{r}=\left\langle\boldsymbol{\varepsilon}_{n}^{p}\right\rangle_{r}+\left\langle\Delta \boldsymbol{\varepsilon}^{p}\right\rangle_{r}, \\
& p^{(r)}=p_{n}^{(r)}+\Delta p^{(r)} .
\end{aligned}
$$

3. Compute the macroscopic stress:

$$
\overline{\boldsymbol{\sigma}}=\overline{\boldsymbol{C}}_{0}: \overline{\boldsymbol{\varepsilon}}^{t r}
$$

The numerical procedure can be combined with any linear MF model suited for the microstructure under consideration and for which an expression for the effective stiffness $\overline{\boldsymbol{C}}_{0}$ is provided. When this expression is available in closed-form (this is the case of the Mori-Tanaka model considered below), the procedure is efficiently implemented using Newton's method. It necessitates the differentiation of the residual function (68) w.r.t. the shear moduli. Some details about the numerical resolution with Newton's method are given in Appendix B together with the macroscopic consistent tangent operator.

\section{Application to two-phase composites}

In this section, the proposed homogenization procedure is used to predict the overall behavior of several elasto-(visco) plastic composites. We focus on two-phase composites presenting a particulate microstructure, with a low to moderate volume fraction of inclusions. For such microstructures, the Mori-Tanaka method is known to give reliable predictions of the overall response of linear composites. The Mori-Tanaka method is thus a natural choice for the homogenization of the LCC. An advantage of the Mori-Tanaka model is that analytical expressions for the effective properties of the LCC are available in closed-form. 
The Mori-Tanaka model (Mori and Tanaka, 1973; Benveniste, 1987) relies on the solution of the equivalent inclusion problem, due to Eshelby (1957). For this model, the strain concentration tensor for the inclusion phase is given by (inclusions and matrix are phases 1 and 2, respectively):

$$
\boldsymbol{A}^{(1)}=\left\{\boldsymbol{I}+c_{2} \boldsymbol{P}:\left(\boldsymbol{C}_{0}^{(1)}-\boldsymbol{C}_{0}^{(2)}\right)\right\}^{-1},
$$

where $\boldsymbol{P}$ is Hill's polarization tensor, which depends solely on the matrix properties and the geometry of the inclusions. According to the well-known result of Eshelby, the strain field within the inclusion predicted by the Mori-Tanaka model is uniform.

The procedure of Section 5 was implemented using Newton's method, as described in Appendix B. In the following examples, usually less than 10 iterations are needed to reach convergence. This number falls to 3 or 4 in the case of elasto-plastic phases. All MF simulations took less than $1 s$ of CPU time on an ordinary PC when subdividing one cycle of uniaxial tension/ compression into 200 time increments. The predictions of the proposed approach are compared to reference results provided by full-field, FE computation on representative cells of the microstructure. The FE results are either original, or gathered from the literature.

\subsection{Dissipation functions}

Before giving numerical examples, we first specify the dissipation functions which will be considered in the sequel.

Norton's power law The kinetic relation for Norton's power law is given by

$$
\dot{p}= \begin{cases}\dot{p}_{0}\left(\frac{\sigma_{v}}{\sigma_{Y}}\right)^{m} & \text { if } \sigma_{e q} \geqslant \sigma_{Y}+R, \\ 0 & \text { otherwise }\end{cases}
$$

where $\sigma_{v} \equiv \sigma_{e q}-R-\sigma_{Y}$ is commonly called the viscous stress. Recalling that $Y=\sigma_{e q}-R$, Norton's power law is obtained choosing the following dissipation potential:

$$
\phi(Y)= \begin{cases}\frac{\dot{p}_{0} \sigma_{Y}}{m+1}\left(\frac{Y-\sigma_{Y}}{\sigma_{Y}}\right)^{m+1} & Y \geqslant \sigma_{Y}, \\ 0 & Y<\sigma_{Y},\end{cases}
$$

or, equivalently:

$$
\phi^{*}(\dot{p})= \begin{cases}\dot{p} \sigma_{Y}+\frac{\dot{p} \sigma_{Y}}{M+1}\left(\frac{\dot{p}}{\dot{p}_{0}}\right)^{M+1} & \dot{p} \geqslant 0, \\ +\infty & \dot{p}<0,\end{cases}
$$

with $M=1 / m$. One can readily check that the kinetic relation (9) yields relation (70).

Perzyna's power law As compared to Norton's law, this viscosity function leads to additional isotropic hardening due to the presence of a drag stress $D(p)$ :

$$
\dot{p}= \begin{cases}\dot{p}_{0}\left(\frac{\sigma_{v}}{D(p)}\right)^{m} & \text { if } \sigma_{e q} \geqslant \sigma_{Y}+R \\ 0 & \text { otherwise. }\end{cases}
$$

The corresponding dissipation function is:

$$
\phi(Y ; p)= \begin{cases}\frac{\dot{p}_{0} D(p)}{m+1}\left(\frac{Y-\sigma_{Y}}{D(p)}\right)^{m+1} & Y \geqslant \sigma_{Y}, \\ 0 & Y<\sigma_{Y},\end{cases}
$$

or, equivalently:

$$
\phi^{*}(\dot{p} ; p)= \begin{cases}\dot{p} Y_{0}+\frac{\dot{p} D(p)}{M+1}\left(\frac{\dot{p}}{\dot{p}_{0}}\right)^{M+1} & \dot{p} \geqslant 0, \\ +\infty & \dot{p}<0 .\end{cases}
$$

The drag stress $D(p)$ can be given a general expression of the form $D(p)=K+\zeta R(p)$ which corresponds to a drag stress proportional to the hardening stress (Chaboche, 2008). Here, Perzyna's approach is adopted (Perzyna, 1964$): K=\sigma_{Y}$ and $\zeta=1$. Note that this power law is implemented in the commercial software ABAQUS 6.9 (2009).

\subsection{Metal Matrix composite}

We start with a Metal Matrix Composite (MMC) consisting of an elasto-plastic matrix reinforced by spherical inclusions. The spatial distribution of the inclusions is random, and two volume fractions of inclusions are considered: $c_{1}=0.15$ and $c_{1}=0.25$. The material properties of the inclusions and matrix are the following: 
- Inclusions (phase 1): $E=400 \mathrm{GPa}, v=0.2$.

- Matrix (phase 2): $E=75 \mathrm{GPa}, v=0.3, \sigma_{Y}=75 \mathrm{MPa}, R(p)=h p^{n}, h=400 \mathrm{MPa}, n=0.4$ or $n=0.05$.

The behavior of the matrix is assumed to be rate-independent at this point. Such properties are representative of an Aluminum matrix reinforced by SiC inclusions. MMC's with similar material properties were previously considered by several authors aiming to assess homogenization models (Segurado et al., 2002; Michel and Suquet, 2003; Doghri and Ouaar, 2003; González et al., 2004; Chaboche et al., 2005; Pierard et al., 2007a) so that the predictive capabilities of the present approach can easily be evaluated w.r.t. those schemes. The predictions of the proposed variational estimate (labeled VAR in the figures) are compared to original FE results obtained on periodic cells containing 35 randomly dispersed inclusions. Details about microstructure generation and FE computation can be found in Brassart et al. (2010). For comparison, we also show results obtained with an incremental tangent method (Doghri and Ouaar, 2003; Pierard and Doghri, 2006b), labeled "Inc.Tgt" in the figures and briefly described in Appendix C.

\subsubsection{Uniaxial tension/compression cycles}

The composites are subjected to uniaxial tension/compression along direction 1 . For $c_{1}=0.15$ (Fig. 3(a)), the variational method correctly predicts the effective response, while the incremental tangent approach yields a slight overestimation. For $c_{1}=0.25$ (Fig. 3(b)), the incremental tangent scheme is slightly more accurate than the variational method, which underestimates the macroscopic stress, except after load reversal. At low hardening exponent, FE results exhibit a significant Bauschinger effect during macroscopic plastic yielding in compression. This effect is not captured by the MF models. The variational method always predicts a symmetric loading/unloading response, as an outcome of the assumption of compatible trial strain. Indeed, the homogenized yield criterion (66) uses the second moment of the trial strain field in the phase computed on the LCC with the actual (constant) elastic properties (cf. the elastic predictor step in Section 5). Given expression (66) for the second moment, it is concluded that reverse plastic yielding occurs when the macroscopic trial strain reaches the same value (in norm) as that of the previous plastic time increment. After the elastic-plastic transition, the

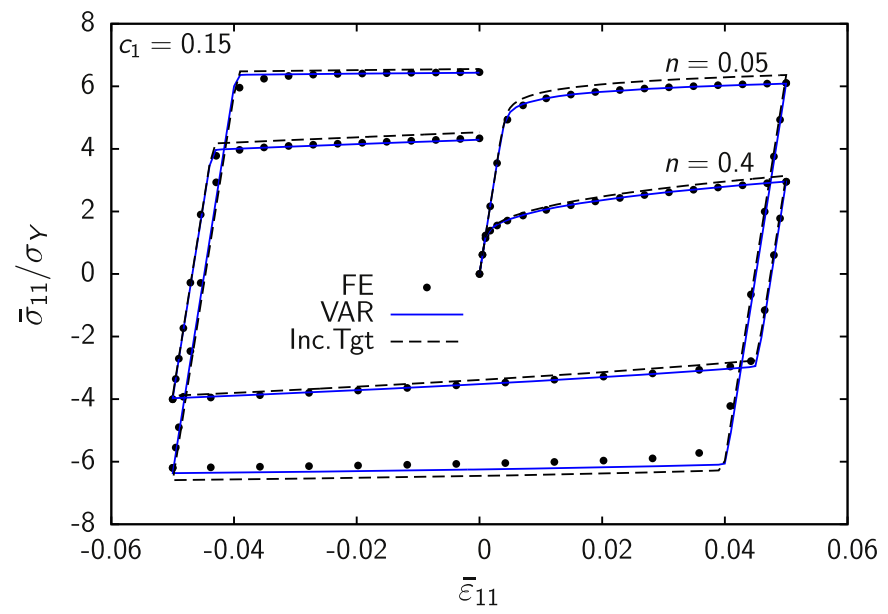

(a)

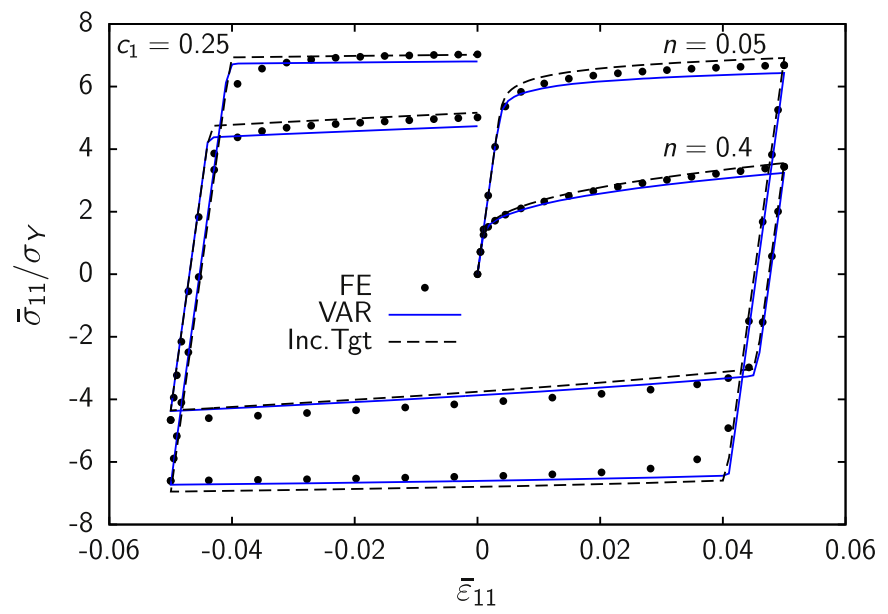

(b)

Fig. 3. Effective response of a metal matrix composite with (a) $15 \%$ and (b) $25 \%$ inclusions volume fraction. Uniaxial tension is applied along direction 1. 


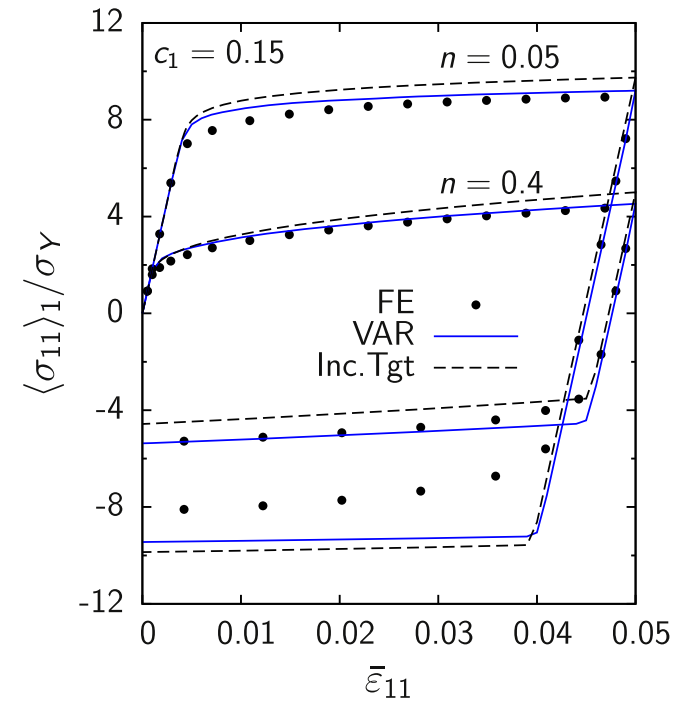

(a)

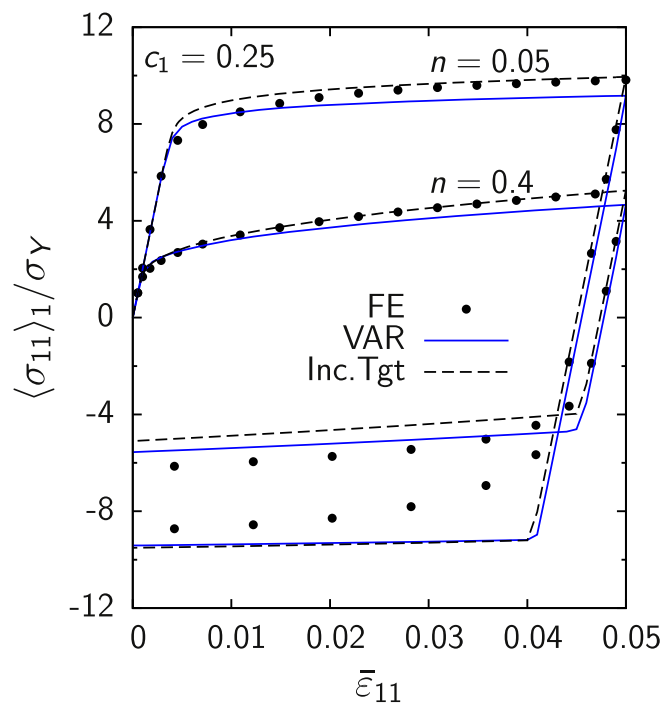

(b)

Fig. 4. Average response of the inclusion phase of a metal matrix composite with (a) $15 \%$ and (b) $25 \%$ inclusions volume fraction.

MF model accounts only for the isotropic hardening of the matrix (and the reinforcing effect of the particles). In contrast, transition from elasticity to plasticity is smoother when predicted by the full-field model. These observations enlighten the limited validity of the assumption of compatibility of the trial strain field. The latter overlooks the plastic strain incompatibilities developing mostly around the particle/matrix interfaces during the abrupt loading path change and which are responsible for the early and progressive plastic yielding observed in the full-field simulation.

Similar observations hold regarding the predictions of the inclusion response (Fig. 4). The largest discrepancy between mean-field and reference results appears during the elastic-plastic transition in compression for the lowest hardening exponent. This, again, may be related to the assumption of compatible trial strain field. Surprisingly, the MF models predict very close inclusion responses for both volume fractions. This is not supported by FE results, which demonstrate a (moderate) increase in the inclusions stress when their volume fraction increases. The inclusion stress level predicted by the variational method is even lower for $c_{1}=0.25$ than for $c_{1}=0.15$. This can be attributed to the decrease of the shear modulus $\mu_{0}^{(2)}$ at higher volume fraction (due to an increase in $\Delta p^{(2)}$, see expression (54)). Indeed, reduced load is then transferred to the inclusions. This unwanted effect does not affect the effective response significantly thanks to the relative volume fractions of phases in the average $\sum c_{r}\langle\boldsymbol{\sigma}\rangle_{r}=\overline{\boldsymbol{\sigma}}$.

\subsubsection{Effect of the triaxiality}

The proposed variational method can sustain general loading conditions, including multiaxial ones. In order to investigate the effect of triaxiality on the accuracy of the predictions, we successively applied a shear (Fig. 5(a)) and a biaxial loading (Fig. 5(b)) to the composite with $c_{1}=0.15$. For shear loading, the only non-vanishing component of the stress tensor is

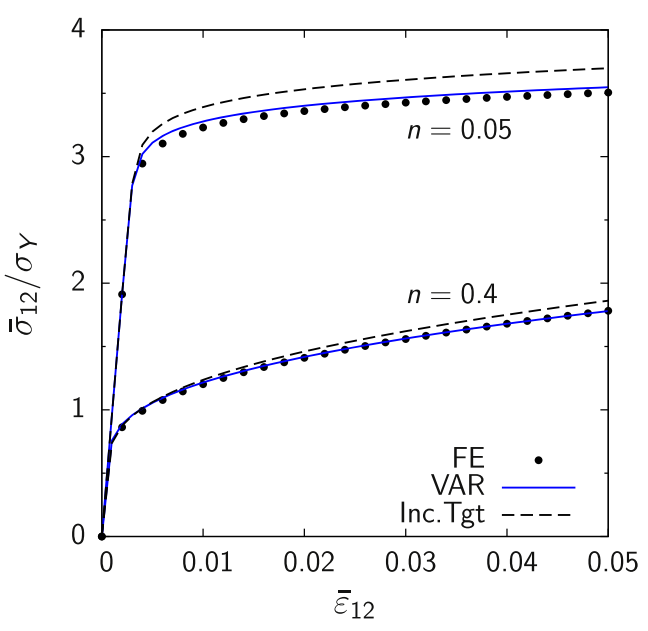

(a)

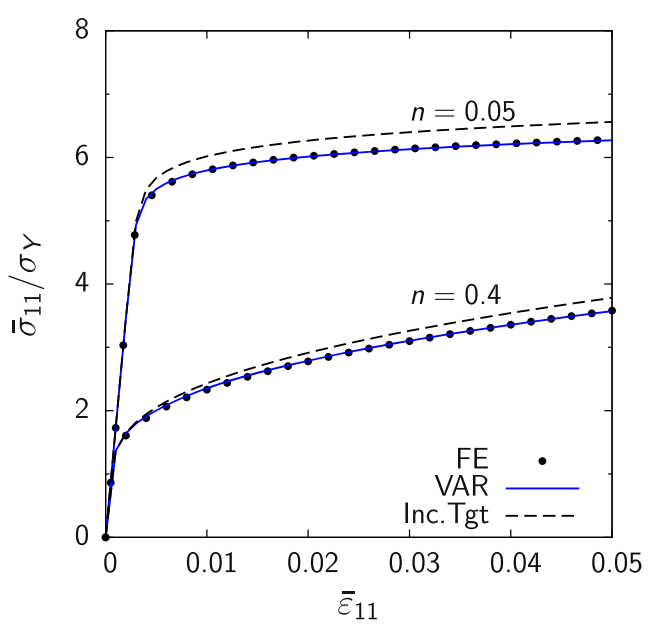

(b)

Fig. 5. Effective response of a metal matrix composite with $15 \%$ of spherical inclusions: (a) composite subjected to shear loading, (b) composite subjected to biaxial tension. 


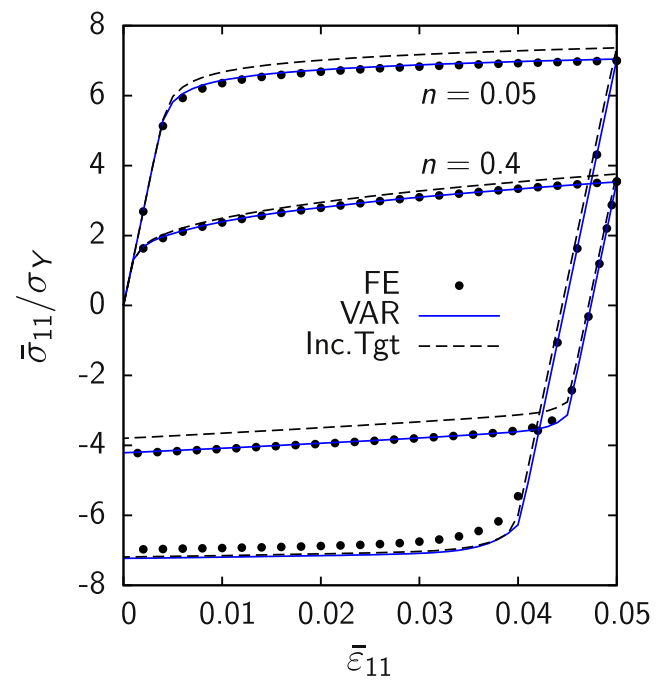

(a)

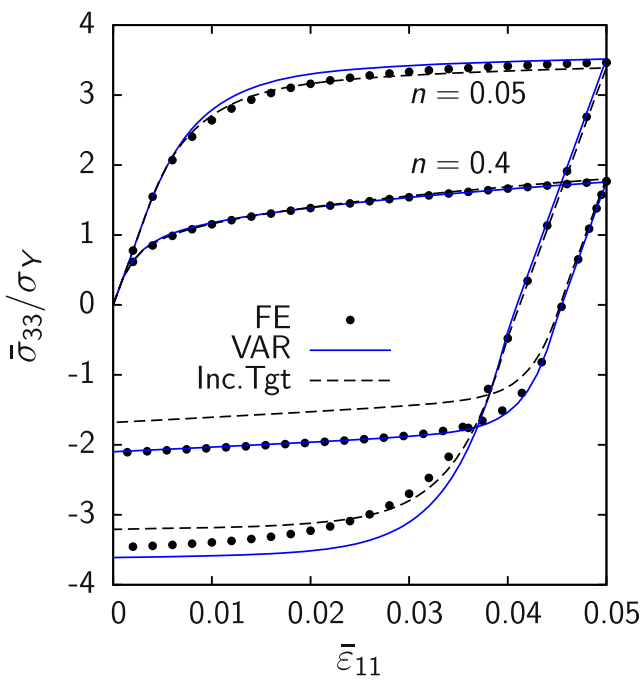

(b)

Fig. 6. Effective response of a metal matrix composite with $15 \%$ of spherical inclusions under a plane strain tension/compression test applied along direction 1 , with $\bar{\varepsilon}_{33}=0$ and $\bar{\sigma}_{22}=0$.

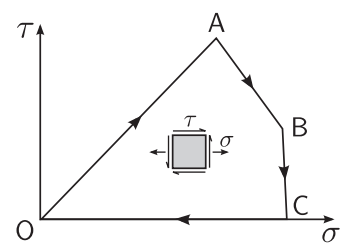

Fig. 7. Non-radial plane stress tension-shear loading, after Nyssen (1981), with $\tau$ the shear stress and $\sigma$ the tensile stress. The loading path is (O-A-B-C-O).

$\bar{\sigma}_{12}=\bar{\sigma}_{21}=\sigma$. Biaxial loading is obtained setting $\bar{\sigma}_{11}=\bar{\sigma}_{22}=\sigma$ and $\bar{\sigma}_{33}=0$. In the principal stress space, the macroscopic triaxiality ratio is given by $\bar{T}=\operatorname{tr}(\overline{\boldsymbol{\sigma}}) / 3 \bar{\sigma}_{e q}$. Shear, uniaxial and biaxial tension correspond respectively to triaxiality ratios of $0,1 / 3$ and $2 / 3$. An excellent agreement with full-field predictions is found in every case.

Then, we consider a plane strain tension/compression test such that the only non-vanishing components of $\bar{\varepsilon}$ are $\bar{\varepsilon}_{11}$ and $\bar{\varepsilon}_{22}$, and $\bar{\varepsilon}_{22}$ is computed such that $\bar{\sigma}_{22}=0$. The stress response of the composite along direction 1 and 3 is shown in Fig. 6 for $c_{1}=0.15$. It corresponds to a triaxiality ratio of approximately 1 in the plastic regime. The variational method provides excellent predictions for the highest hardening exponents, and gives very satisfying predictions for the lowest one. Again, the major discrepancy is observed during yielding in compression. In all these examples, the new method performs better than the incremental tangent approach.

Finally, we consider a non-monotonic, non-proportional loading path. The stress state is such that the only non-vanishing stress components are $\bar{\sigma}_{11}=\sigma$ and $\bar{\sigma}_{12}=\tau$. This plane stress state combining tension and shear can represent the stress in a thin walled cylindrical tube under axial tension and torsion, far from the ends. The non-proportional loading path was proposed by Nyssen (1981) as a benchmark for testing nonlinear computing codes and is depicted in Fig. 7. Here, we adopted the following values of $(\sigma, \tau)$ at $O, A, B$ and $C$ (in MPa):

$$
O(0,0), \quad A(150,75), \quad B(206.3,37.5), \quad C(210,0) .
$$

The stress state at $B$ was chosen such that the segment $A-B$ is approximately tangent to the yield surface at $A$ of the composite with $c_{1}=0.15$ and $n=0.4$. The unknown yield surface was identified from the reference solution for the composite under uniaxial tension (Fig. 3(a)), assuming an isotropic response. The comparison between reference and MF results obtained with the proposed method is reported in Fig. 8 for the composite with $c_{1}=0.15$ and $n=0.4$. The MF predictions are very satisfying, except during the early stages of the first path change. This example also illustrates the robustness of the proposed formulation and algorithm.

\subsubsection{Ellipsoidal inclusions}

We now consider a MMC reinforced by ellipsoidal inclusions. The inclusions are aligned and have an aspect ratio $\alpha=3$. Other material properties are the same as those used for spherical inclusions, and the volume fraction is set to $c_{1}=0.15$. The composites are subjected to a uniaxial tension/compression test. The loading is applied either along the direction of the fibers (Fig. 9(a)) or in the transverse direction (Fig. 9(b)). The new method gives significantly better predictions than the incremental tangent approach when loading is applied in the longitudinal direction. The overestimation of the 


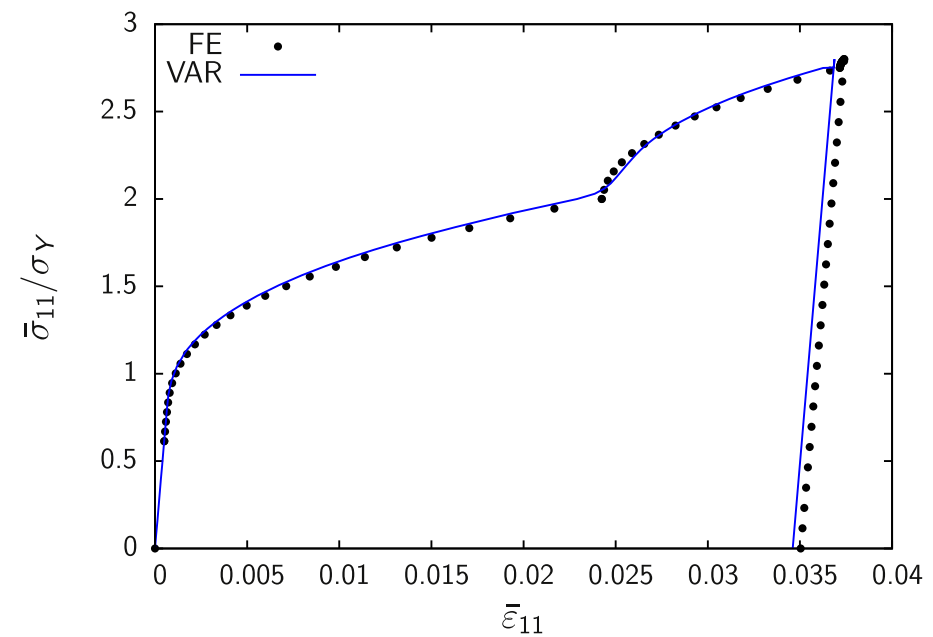

(a)

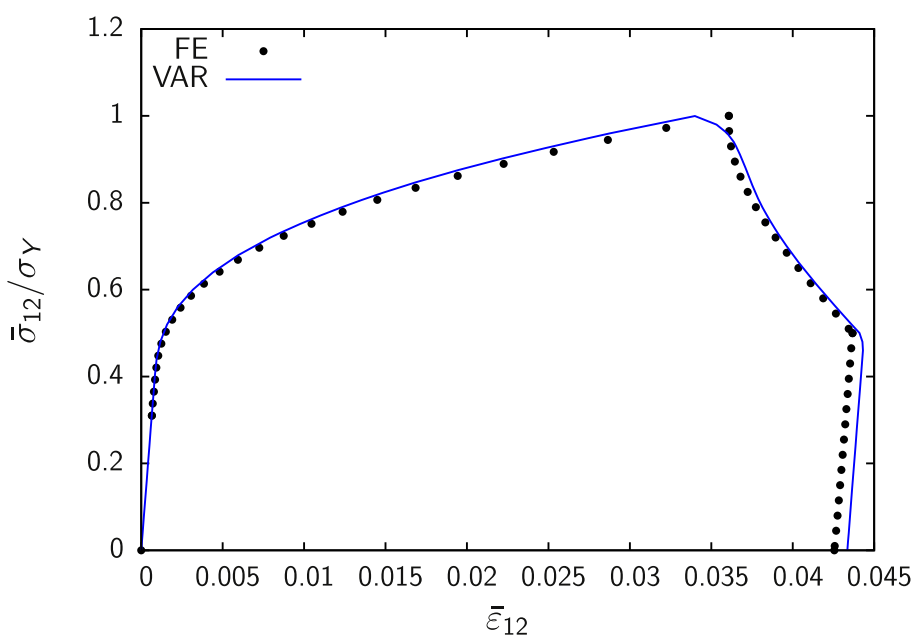

(b)

Fig. 8. Effective response of a metal matrix composite with $15 \%$ of inclusions subjected to non-radial plane stress tension-shear loading. The matrix hardening exponent is $n=0.4$.

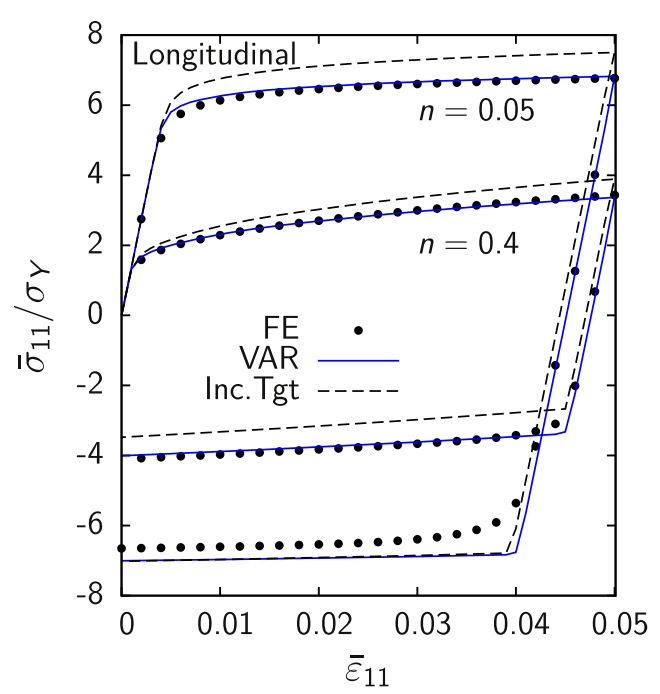

(a)

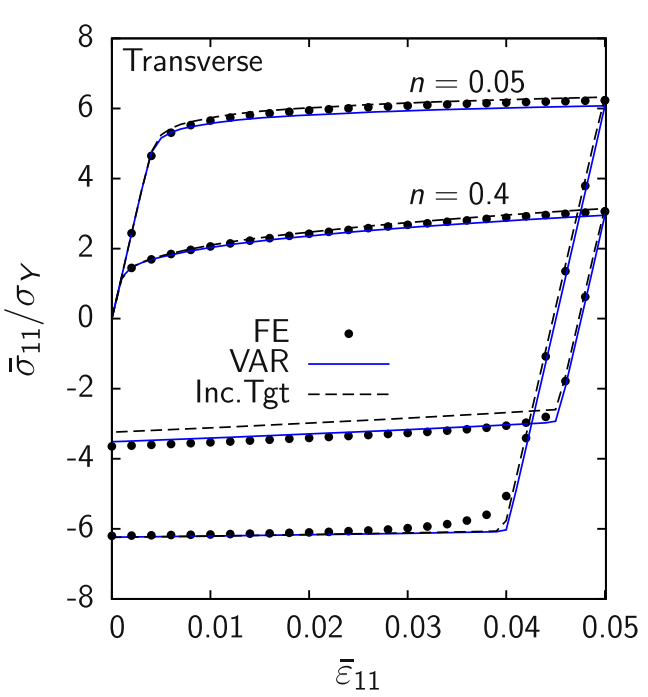

(b)

Fig. 9. Effective response of a metal matrix composite with $15 \%$ of aligned, ellipsoidal inclusions under uniaxial tension/compression. Tension is applied either along the direction of the fibers (a) or in the transverse direction (b). 
incremental tangent approach was previously reported by Pierard et al. (2007a). These authors attributed the discrepancy between the incremental tangent and the full-field results to the large strain heterogeneities taking place between the ends of the elongated ellipsoids and which are particularly important when the strain hardening exponent is low. The strain heterogeneities are overlooked by the incremental approach, while they are somehow included in the variational method through the second moments of the trial strain, which might explain the better performance of the latter in this case. Strain heterogeneities are less important when loading is applied in the transverse direction, and both the incremental tangent method and the proposed model yield then satisfying predictions.

\subsubsection{Rate-dependent case}

We now consider the case of a rate-dependent behavior of the matrix. Norton's power law (70) is considered, taking $\dot{p}_{0}=0.01 \mathrm{~s}^{-1}$ and $m=5$. The volume fraction of inclusions is $c_{1}=0.15$, and the hardening exponent for the matrix is $n=0.4$. All other material parameters are the same as those used for rate-independent simulations. Predictions of the proposed model are compared to FE results and to predictions of the incrementally affine model of Doghri et al. (2010). The latter was implemented in the DIGIMAT-MF software (DIGIMAT, 2011) and is briefly described in Appendix D. Corresponding results are labeled "Inc.Aff." in the figures.

Fig. 10 shows the effective response of the composite under uniaxial tension at several macroscopic strain rates. An excellent agreement between FE and mean-field results is found at all strain rates. Surprisingly, the variational and incrementally affine methods give almost identical predictions. A relaxation test is presented in Fig. 11. Uniaxial tension is first performed

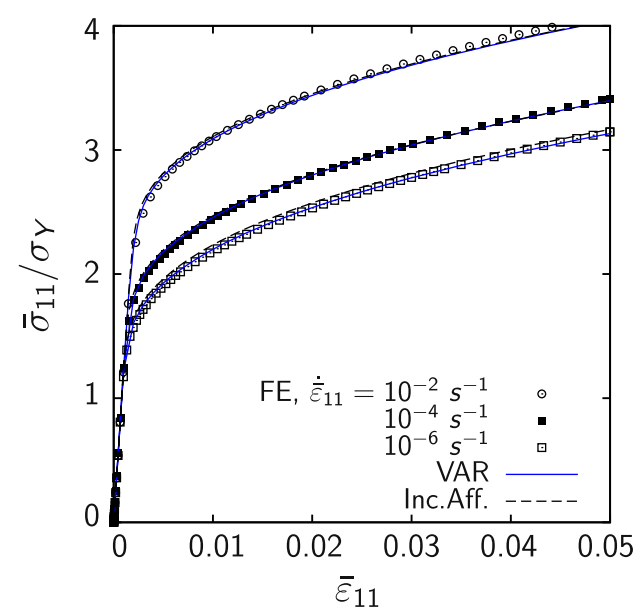

Fig. 10. Effective response of a metal matrix composite with a rate-dependent matrix behavior described by Norton's power law, with rate-sensitivity exponent $m=5$. Uniaxial tension is performed at three different macroscopic strain rates.

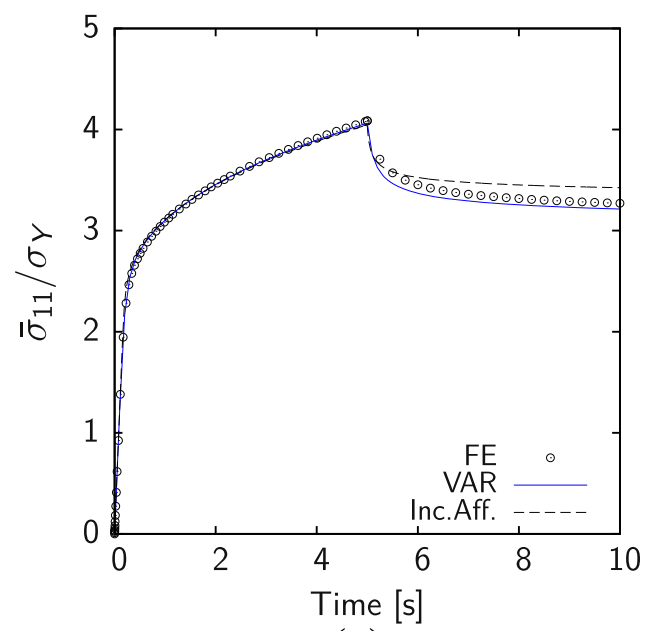

(a)

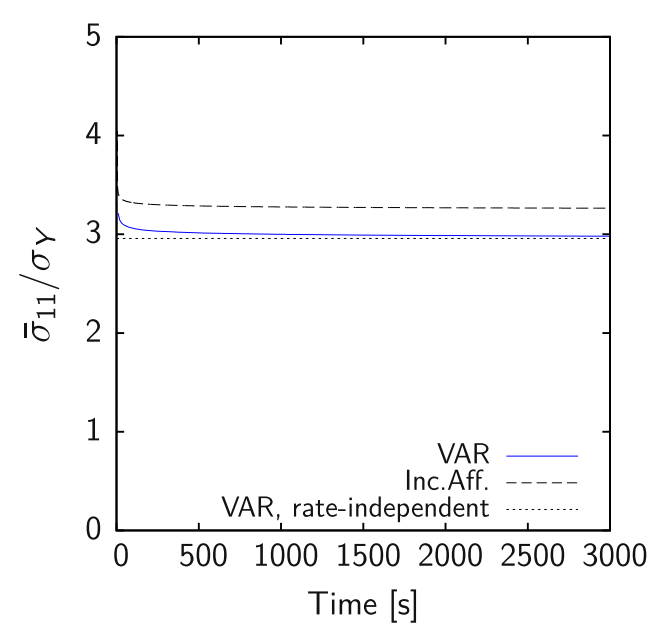

(b)

Fig. 11. Uniaxial tension test up to $\bar{\varepsilon}_{11}=5 \%\left(\dot{\bar{\varepsilon}}_{11}=0.01 \mathrm{~s}^{-1}\right)$ followed by a relaxation test maintaining the overall strain constant. The matrix rate-sensitivity exponent is $m=5$. Short-term (a) and longer-term (b) response of the composite. 


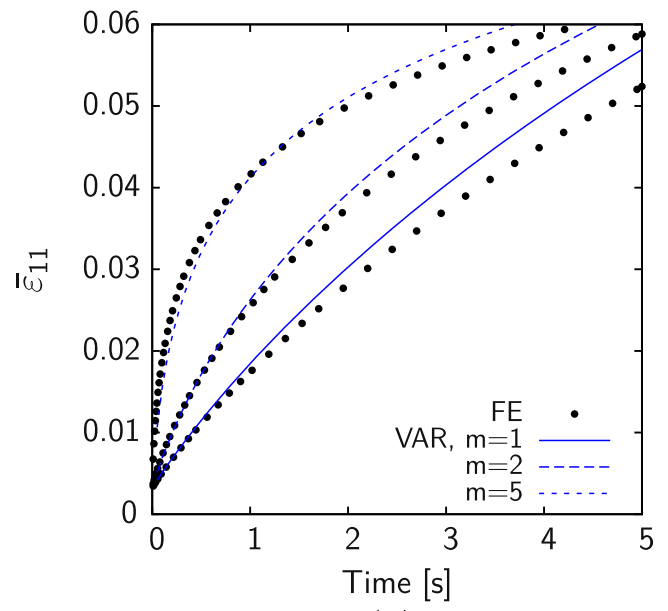

(a)

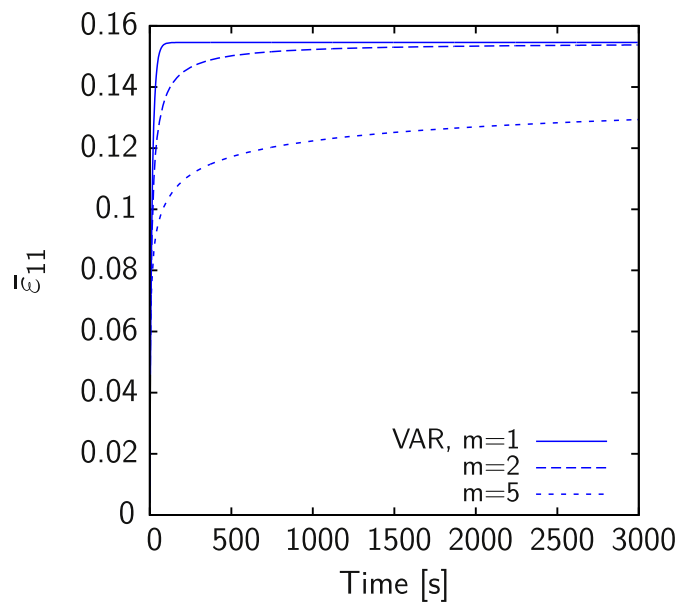

(b)

Fig. 12. Creep test under instantaneous, constant uniaxial load, with $\bar{\sigma}_{11}=4 \sigma_{Y}$. Three matrix rate-sensitivity exponents are considered: $m=5$, $m=2$ and $m=1$. Short-term (a) and longer-term (b) response of the composites.

at a strain rate $\dot{\overline{\boldsymbol{\varepsilon}}}_{11}=0.01 \mathrm{~s}^{-1}$ up to $5 \%$ elongation. The final elongation is then maintained, while the stress relaxes. While the variational and incrementally affine models give almost identical responses for the initial uniaxial tension test, their responses significantly deviate during stress relaxation (Fig. 11(a)). The new model is then more accurate than the incrementally affine model. After a sufficiently long relaxation time, the macroscopic stress predicted by the variational method tends to its rate-independent limit, as expected (Fig. 11(b)).

Finally, the response of the composite under creep is presented in Fig. 12. Creep is achieved by applying a instantaneous macroscopic stress $\bar{\sigma}_{11}=4 \sigma_{Y}$, while the $\bar{\sigma}_{22}$ and $\bar{\sigma}_{33}$ components are zero. Three rate-sensitivity exponents are considered: $m=1, m=2$ and $m=5$. The loading time is about an hour. The response of the composite in the early stages of creep are shown in Fig. 12(a). The proposed model correctly predicts the main trends of the response, but slightly overestimates the creep strain rate, so that the predicted macroscopic strain deviates from the reference strain. The long-term trends of the response as predicted by the proposed model are shown in Fig. 12(b). After $3000 \mathrm{~s}$, the creep responses for $m=1$ and $m=2$ have reached stationary values, while the creep strain is still increasing in the case $m=5$.

\subsection{Elasto-viscoplastic matrix reinforced by elasto-viscoplastic inclusions}

We now consider a composite with two elasto-viscoplastic phases previously studied by Pierard et al. (2007b). Again, inclusions are spherical and randomly distributed within the continuous matrix. Both phases present isotropic hardening described by the power law $R(p)=h p^{n}$, and viscosity function of the Perzyna-type (73), with $\operatorname{drag} \operatorname{stress} D(p)=\sigma_{Y}+R(p)$. The material properties are the following:

- Inclusions: $E=400 \mathrm{GPa}, v=0.286, \sigma_{Y}=400 \mathrm{MPa}, h=8 \mathrm{GPa}, n=0.4, \dot{p}_{0}=210^{-4} \mathrm{~s}^{-1}$ and $m=1.5$.

- Matrix: $E=70 \mathrm{GPa}, v=0.33, \sigma_{Y}=70 \mathrm{MPa}, h=4 \mathrm{GPa}, n=0.4, \dot{p}_{0}=310^{-4} \mathrm{~s}^{-1}$ and $m=1.5$.

Two inclusions volume fractions are considered: $c_{1}=0.15$ and $c_{1}=0.30$. Reference FE predictions were obtained by Pierard et al. (2007b), and details about the microstructure generation and FE meshes can be found in their paper. Next to the predictions of the variational approach, results obtained with the incrementally affine method (see Appendix D) are also presented.

The effective response of the composite undergoing uniaxial tension at several macroscopic strain rates is presented in Fig. 13. In every case, predictions of the variational method and reference results agree. The model captures both the effect of the strain rate, and the effect of the volume fraction of inclusions. In this example, the variational method performs better than the incrementally affine method.

Phase responses for $c_{1}=0.3$ are presented in Fig. 14 , for $\dot{\bar{\varepsilon}}_{11}=10^{-3} \mathrm{~s}^{-1}$ and $\dot{\bar{\varepsilon}}_{11}=10^{-6} \mathrm{~s}^{-1}$. The reference result is the average of the von Mises stress computed from the FE results according to:

$$
\left\langle\sigma_{e q}\right\rangle_{r}=\left(\sum_{k}\left(\sigma_{e q, k} V_{k}^{(r)}\right)\right) /\left(\sum_{k} V_{k}^{(r)}\right)
$$

where $\sigma_{e q, k}$ is the von Mises stress at the $k$ th integration point in phase $r$, and $V_{k}^{(r)}$ the associated volume. As the average von Mises stress is not directly available from the MF models, first- and second-order measures are considered instead. The firstorder measure is obtained by simply taking the von Mises norm of the average stress in each phase: 


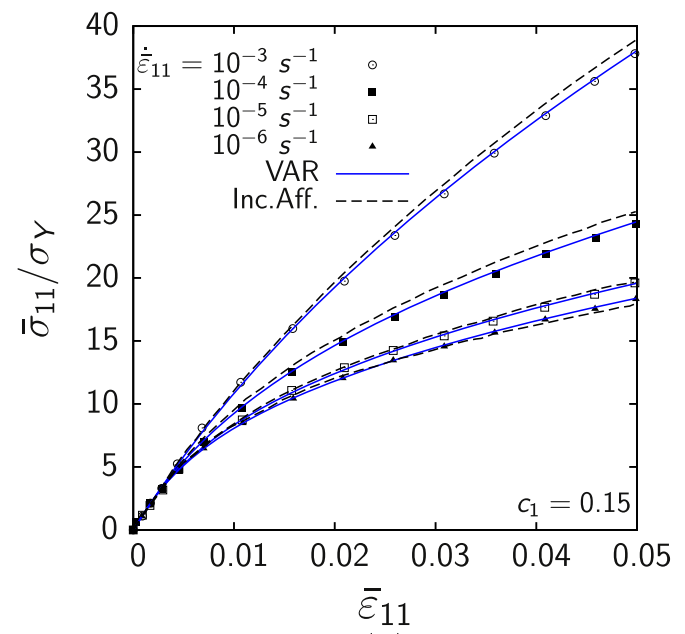

(a)

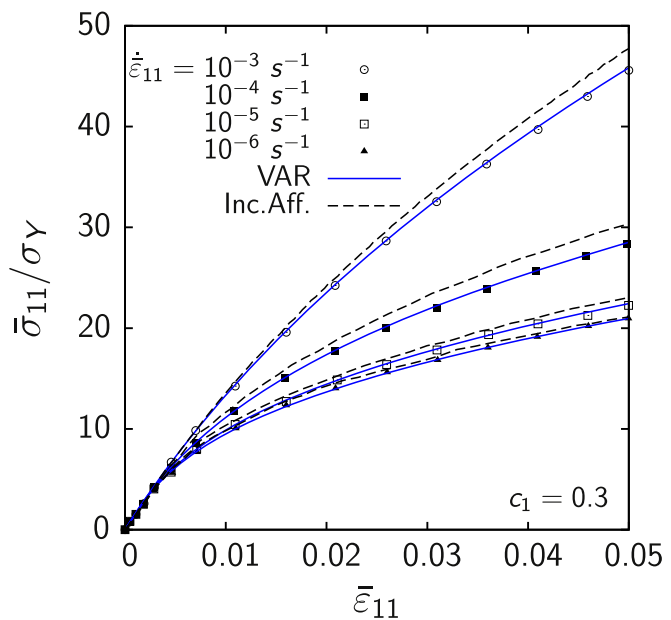

(b)

Fig. 13. Effective response of a composite with elasto-viscoplastic inclusions and matrix, for (a) $15 \%$ and (b) $30 \%$ of inclusions volume fraction.

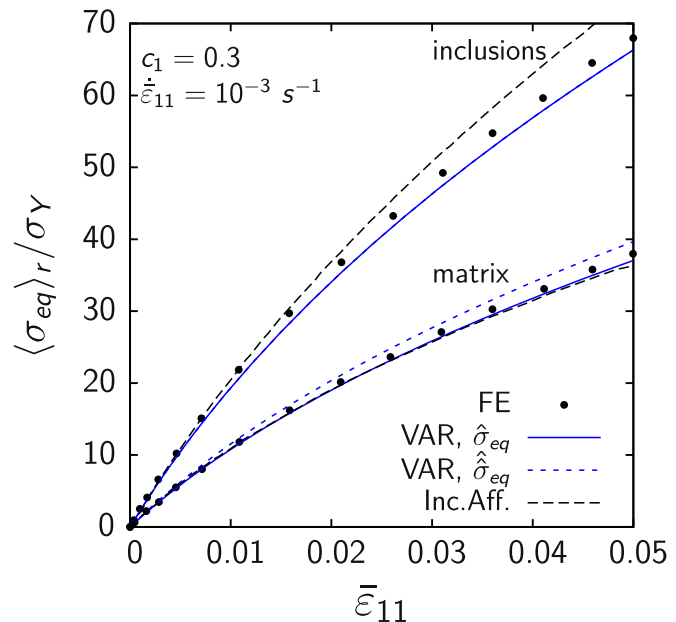

(a)

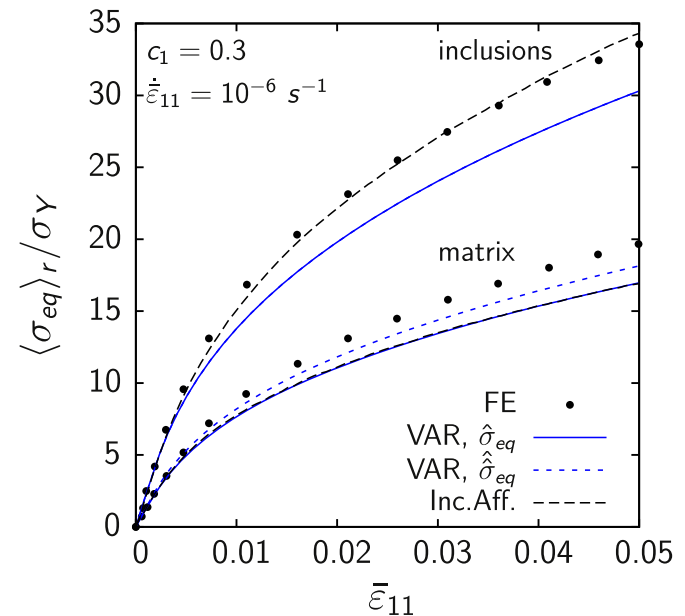

(b)

Fig. 14. Phase response of a composite with elasto-viscoplastic inclusions and matrix, for $30 \%$ of inclusions volume fraction. The applied macroscopic strain rate is (a) $\dot{\bar{\varepsilon}}_{11}=10^{-3} \mathrm{~s}^{-1}$ and (b) $\dot{\bar{\varepsilon}}_{11}=10^{-6} \mathrm{~s}^{-1}$.

$$
\hat{\sigma}_{e q}^{(r)} \equiv\left(\langle\boldsymbol{\sigma}\rangle_{r}\right)_{e q}=3 \mu_{0}^{(r)}\left(\left\langle\varepsilon^{t r}\right\rangle_{r}\right)_{e q}
$$

and the second-order measure is given by

$$
\hat{\hat{\sigma}}_{e q}^{(r)} \equiv 3 \mu_{0}^{(r)} \sqrt{\left\langle\left(\varepsilon_{e q}^{t r}\right)^{2}\right\rangle_{r}} .
$$

The incrementally affine method yields only a first-order measure. Predictions of the proposed scheme are satisfying at the highest strain rate, but underestimate the stress level at low strain rate sensitivity. Predictions of the incrementally affine method are then closer to reference curves.

The effect of the triaxiality is investigated in Fig. 15 which shows the prediction of the models for the composite subjected to shear and biaxial tension. Although the accuracy of the variational method under shear is very good at the lowest strain rate, it significantly decreases when the strain rate increases, the variational method underestimating then the reference response (Fig. 15(a)). On the other hand, the proposed method remains accurate for all considered loading rates under biaxial tension (Fig. 15(b)).

Finally, results for one cycle of tension/compression are presented in Fig. 16 for $\dot{\bar{\varepsilon}}_{11}=10^{-3} \mathrm{~s}^{-1}$ and $\dot{\bar{\varepsilon}}_{11}=10^{-6} \mathrm{~s}^{-1}$, with $c_{1}=0.30$. As in the monotonic case, the predictions of the variational approach and reference results agree. 


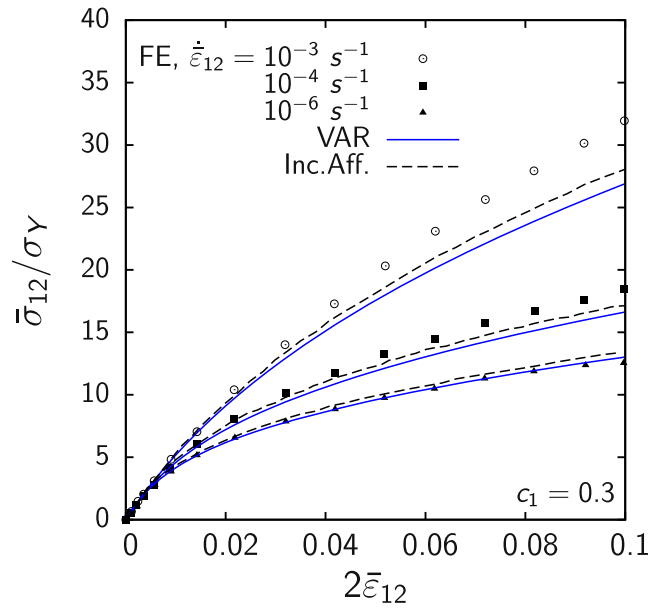

(a)

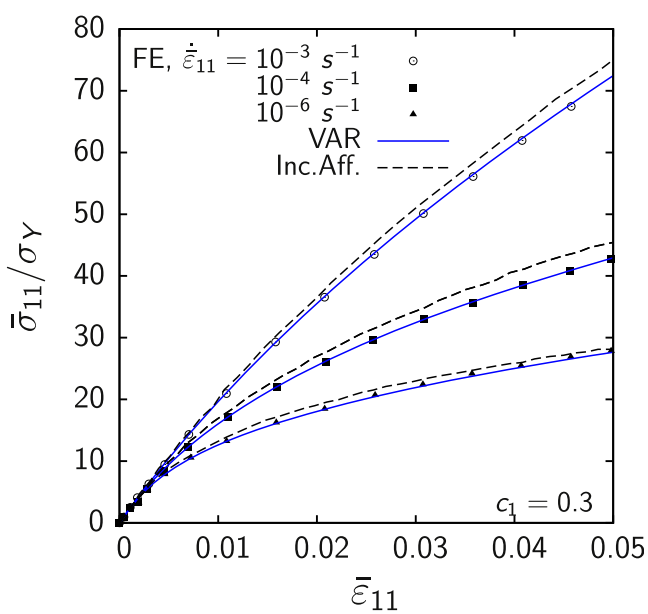

(b)

Fig. 15. Effective response of the composite with $c_{1}=0.3$ under shear loading (a) and biaxial tension (b).

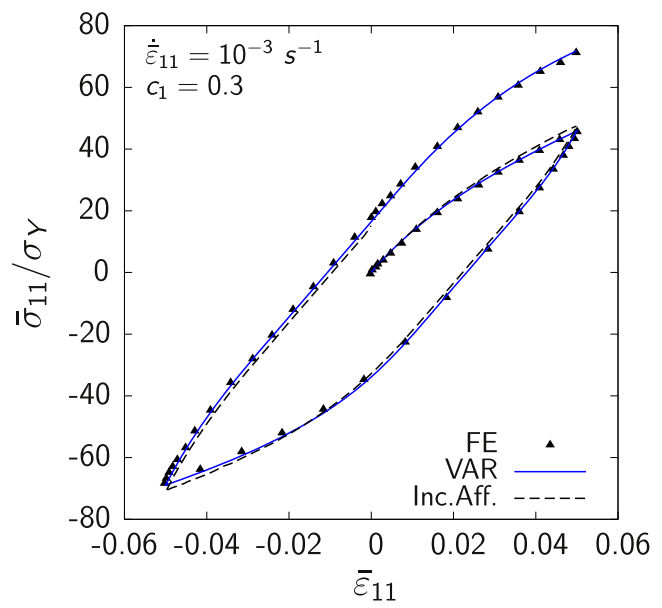

(a)

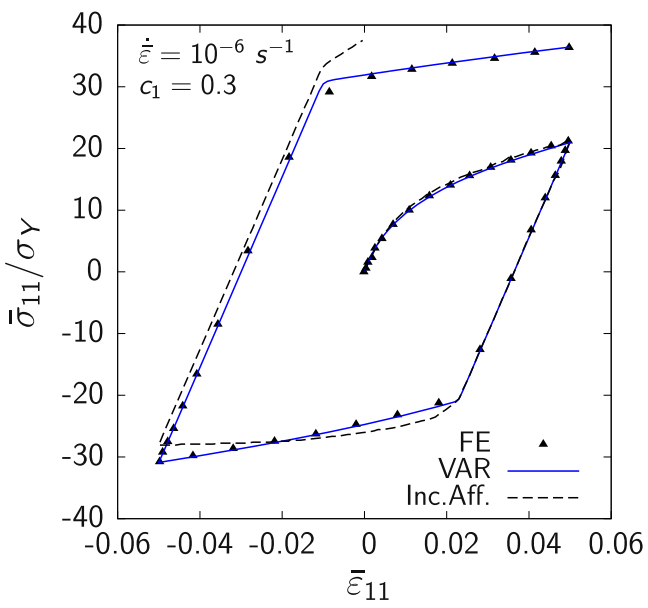

(b)

Fig. 16. Effective response of the composite with $30 \%$ of inclusions under one cycle of tension $/$ compression, for $\left(\right.$ a) $\dot{\bar{\varepsilon}}_{11}=10^{-3} \mathrm{~s}^{-1}$ and (b) $\dot{\bar{\varepsilon}}_{11}=10^{-6} \mathrm{~s}^{-1}$.

\subsection{Short glass fiber-reinforced polyamide}

Finally, we consider a composite made of a polyamide matrix reinforced by short glass fibers, with the following material properties:

- Inclusions: $E=72 \mathrm{GPa}, v=0.22$.

- Matrix: $E=2.1 \mathrm{GPa}, v=0.3, \sigma_{Y}=29 \mathrm{MPa}$.

The hardening of the matrix material is represented by a linear-exponential law:

$$
R=h_{1} p+h_{2}(1-\exp (-n p))
$$

with $h_{1}=139 \mathrm{MPa}, h_{2}=32.7 \mathrm{MPa}$ and $n=319$. The inclusions are ellipsoidal with aspect ratio $\alpha=15$, and their volume fraction is $c_{1}=0.157$. This composite was proposed as benchmark by Doghri et al. (2011), as the first moment-based incremental tangent method yields poor predictions. In that paper, an incremental tangent method enriched with second-order moments is proposed, which dramatically improves the predictions on this example. Reference results were obtained on prismatic cells containing a large number of aligned sphero-cylinders. ${ }^{2}$ FE simulations were performed using ABAQUS and the DIGIMAT-FE software (DIGIMAT, 2011) and are described in (Doghri et al., 2011). The predictions of the variational approach are compared to those of the first- and second-moment incremental tangent schemes.

\footnotetext{
${ }^{2}$ In the mean-field models, fibers are approximated by ellipsoids. Thus, FE and mean-field predictions do not correspond exactly to the same microstructure.
} 


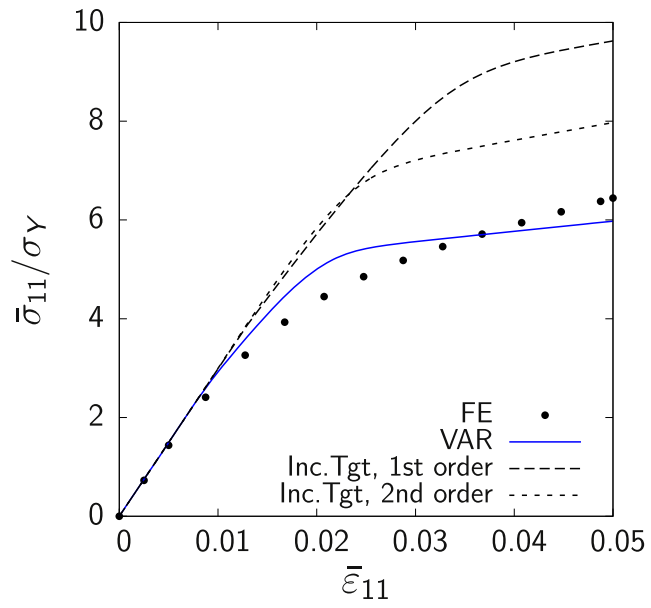

(a)

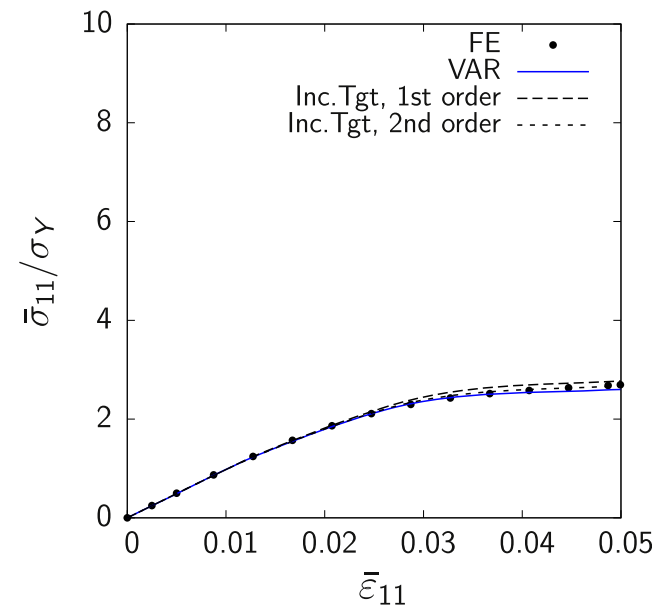

(b)

Fig. 17. Effective response of a short glass fibers-reinforced polyamide under uniaxial tension. The volume fraction of fibers is $15.7 \%$ and their aspect ratio $\alpha=15$. Tension is performed either in the direction of the fibers axes (a) or transversely to the fibers axes (b). The matrix is elasto-plastic, and the fibers are linear elastic.

We consider first a matrix with rate-independent behavior. The composite is subjected to uniaxial tension either in the longitudinal or the transverse direction w.r.t. the fibers revolution axis. The predictions of the effective behavior provided by the MF models and the FE results for the two loading cases are presented in Fig. 17. The behavior of the matrix material without reinforcement is also shown for comparison. When the loading is applied in the longitudinal direction, both incremental tangent methods overestimate the macroscopic stress, but the second-moment procedure significantly improves the predictions (Fig. 17(a)). However, results of the variational method are even closer to the reference curve. In the case of uniaxial tension applied transversely to the direction of the fibers, all MF models give similar and satisfying predictions. Note however that the overall stress level is much lower than in the longitudinal case.

This example is investigated further adding a rate-dependence of the matrix behavior. The Perzyna-type viscous law (73) is considered, with drag stress $D(p)=\sigma_{Y}+R(p)$. The viscous parameters are $\dot{p}_{0}=2.5 \mathrm{~s}^{-1}$ and $m=5$. The MF models considered here are the incrementally affine method and the variational method. Fig. 18 shows the effective response under uniaxial tension applied in the longitudinal direction w.r.t. the fibers axes. As expected, the incrementally affine method overestimates the effective response, as it uses first-order measure of fields only. It can be checked that the curve at the lowest strain rate is very close to the result obtained with the first-order incremental tangent method in the rate-independent case. On the other hand, the variational method is closer to the actual macroscopic stress level, but fails in predicting the slope of the reference curves in the plastic regime, especially at high strain rate.

Finally, the creep response of the short glass fibers-reinforced Polyamide is presented in Fig. 19. The creep test is simulated by applying an instantaneous and constant macroscopic stress $\bar{\sigma}_{11}$, cancelling the other components of the stress tensor. Three stress levels are successively considered: $\bar{\sigma}_{11}=4 \sigma_{Y}, 6 \sigma_{Y}$ and $8 \sigma_{Y}$. For the lowest applied stress, the macroscopic strain rapidly reaches its steady state value. The latter remains close to the instantaneous elastic response of the composite

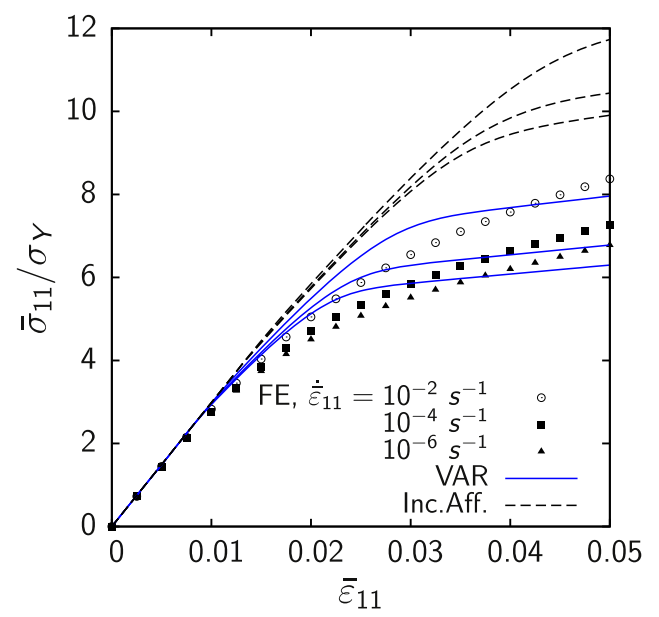

Fig. 18. Effective response of a short glass fibers-reinforced polyamide under uniaxial tension applied in the longitudinal direction w.r.t. the fibers axes. The matrix has an elasto-viscoplastic behavior of the Perzyna-type with rate-sensitivity exponent $m=5$. 


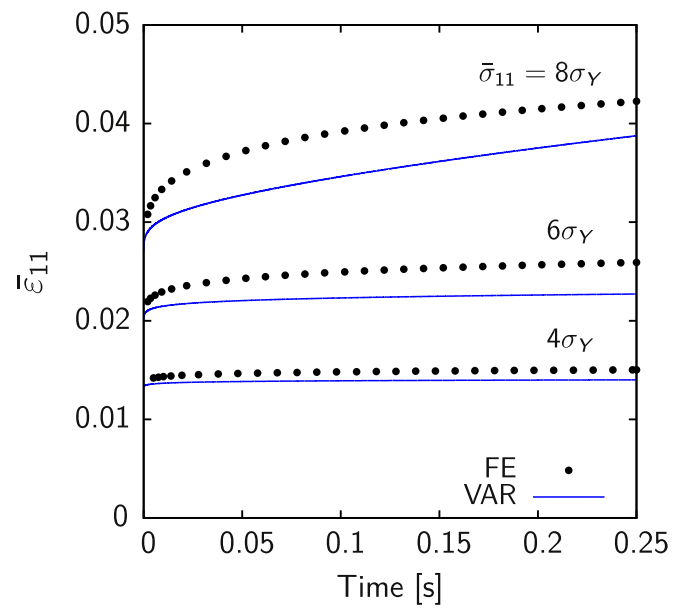

Fig. 19. Creep response of a short glass fibers-reinforced polyamide under a constant load $\bar{\sigma}_{11}=4 \sigma_{Y}, 6 \sigma_{Y}$ or $8 \sigma_{Y}$ (uniaxial tension) applied in the longitudinal direction w.r.t. the fibers axes. The matrix has an elasto-viscoplastic behavior of the Perzyna-type with rate-sensitivity exponent $m=5$.

(for $t=0$ ). The MF model then yields a satisfying prediction, consistently with the trends at low strain observed in Fig. 18 . However, the MF model significantly underestimates the macroscopic strain for higher level of macroscopic stress. This is to be related to the discrepancy between MF and FE predictions after the elastic-plastic transition in Fig. 18. The reasons for the inaccuracy of the proposed MF model in the present example (as compared for instance to the example of the MMC of Section 6.2) are probably related to the much higher constrast between the properties of the constituents, and the high aspect ratio of the fibers. These factors contribute to the development of high field heterogeneities within the composite. In our experience, composites similar to the present short-glass fibers-reinforced thermoplastic constitute some of the most challenging problems for assessing the predictive capabilities of MF theories.

\section{Conclusions}

We presented an original variational formulation for the homogenization of composites having elasto-(visco) plastic constituents. The model is developed within a time-discretized framework, and the past history of deformation is accounted for through internal variables. Both rate-dependent and rate-independent plasticity are handled within a common thermodynamic framework, the only change arising from the specific choice of the dissipation function. The formulation relies on the concept of Linear Comparison Composite (LCC) to be defined at each time step. The effective response of the LCC coincides with the sought-after effective response of the nonlinear composite. An advantage of such formulation is that any homogenization scheme can be used to solve the LCC problem, depending on the microstructure under consideration.

The LCC is characterized by secant operators relating the stress to the elastic predictor strain. These operators are naturally isotropic and softer than corresponding elastic operators. Therefore, isotropy needs not to be prescribed as an heuristic correction, like in incremental tangent methods (Doghri and Ouaar, 2003; Pierard and Doghri, 2006b) and in the incrementally affine model (Doghri et al., 2010). To our knowledge, linearization strategies based on such operators have first been proposed in Brassart et al. (2011). In addition, these operators are computed for the second-order moments of the trial strain field. This, again, results from the variational formulation. Updates of the internal variables obey a homogenized yield criterion and radial return condition. Thus, the algorithmic structure of the incremental equations of elasto-(visco) plasticity is preserved in the homogenization procedure.

The model relies on the following two fundamental assumptions:

- Piecewise uniformity of the comparison moduli $\boldsymbol{C}_{0}^{(r)}$. This assumption also involves piecewise uniformity of the internal variable $p$.

- Compatibility of the trial strain field. Local plastic incompatibilities are ignored, but a macroscopic pre-deformation is applied to the LCC to account for plastic deformation on average at the level of the composite.

The first approximation seems natural, and is commonly adopted in variational formulations for nonlinear composites (like the variational procedure of Ponte Castañeda (1991)). It actually delivers a lower bound on the effective incremental potential, see Eq. (48). Unfortunately, the second approximation has no variational character and its theoretical justification is unclear. Only the valid predictions obtained so far for a variety of composites indicate that the proposed approximation seems reasonable to a certain extent. In addition, this assumption greatly simplifies the homogenization problem of the LCC, as standard schemes for linear composites can readily be applied. It is worth emphasizing that the numerical cost associated with the model is low, so that it is suited for real-scale simulations of composite structures.

The proposed model has been successfully validated in several examples of two-phase composites made of inclusions dispersed in an elasto-(visco) plastic matrix. In all cases, the Mori-Tanaka scheme was used to homogenize the LCC. 
Predictions were systematically compared to reference results obtained from FE computation on multiparticle cells representative of the microstructure. These examples demonstrated that the new variational method competes with other incremental schemes, namely the first- (Doghri and Ouaar, 2003) and second-moment (Doghri et al., 2011) incremental tangent schemes in elasto-plasticity and the incrementally affine method (Doghri et al., 2010) in elasto-viscoplasticity. In all examples considered so far, the variational method yields softer predictions than the incremental tangent method. Consequently, the variational method is likely to perform better than the incremental tangent method in cases where the latter is too stiff. We also emphasize that the new method does not require the heuristic correction of isotropized tangent operators. Moreover, it applies equally well to rate-independent and rate-dependent elasto-viscoplasticity, contrarily to most existing mean-field formulations. The proposed model still needs to be verified for other types of microstructures. For instance, it could be combined with the self-consistent scheme for the modeling of the effective behavior of multiphase aggregates. This study is left for future work.

Comparison of mean-field predictions with FE results on multiparticle cells enlightened (at least) two important trends in the effective response that mean-field models fail to reproduce:

- The inclusions response. MF models performed poorly at the inclusion level in numerous examples. FE results show a progressive stress build-up in the inclusions during plastic yielding of the matrix, while mean-field models predict an abrupt slope change, similar to the slope change in the matrix response during elastic-plastic transition;

- Bauschinger's effect after load reversal. In MF models, elasto-(visco) plastic phases are either fully elastic, or fully plastic. Therefore, they cannot capture progressive plastic yielding related to stress heterogeneities.

The proposed model allows the use of a large variety of (nonlinear) isotropic hardening and viscosity functions. Recently, incremental variational principles have been proposed which also account for nonlinear kinematic hardening (Mosler, 2010; Canadija and Mosler, 2011). They rely on the introduction of additional strain-like internal variables and different expressions for the dissipation function. However, it is not clear whether the proposed homogenization procedure could be generalized to account for kinematic hardening. Indeed, the linearization procedure described in Section 3 cannot directly be applied in the presence of back stresses. This certainly constitutes a drawback of the present formulation compared to, for instance, Hill's incremental approach, where the plasticity model can be used as a black box and local equations are decoupled from the linearization. On the other hand, the proposed linearization technique could probably be generalized to account for compressible plastic flow, provided that the latter can still be expressed in terms of the trial strain within the discretized setting, see for instance Weinberg et al. (2006). In that paper, the authors introduced kinematic constraints of the form (5) for both the deviatoric and hydrostatic part of the plastic strain rate. In this case, the linearization procedure would aim to introduce comparison bulk and shear moduli, which can be performed following a similar variational procedure as in Section 3, see for instance Ponte Castañeda and Suquet (1998). Such generalization of the homogenization model is left for a future work.

Among possible improvements of the presently proposed variational method, one should focus on proposing an alternative technique to solve the LCC problem without violating kinematic restrictions. Indeed, as the assumption of compatibility of the trial strain field is against theoretical and physical evidence, the predictive capabilities of the method cannot be ensured in general. Brassart et al. (2011) presented the pathological example of perfectly plastic composites with perfectly plastic inclusions, but other critical examples could perhaps be found. Tentative research along this way can be found in Brassart (2011), considering piecewise uniform, reference plastic strain.

To conclude, potential-based approaches offer a very rich framework for the development of advanced mean models. In this work, we have proposed a variational technique leading to an estimate (still involving a heuristic prescription). Lahellec and Suquet (2007a,b) proposed a different approach starting from a similar representation of the homogenization problem. Most certainly, there is plenty of room for alternative formulations. In this context, recent progresses in nonlinear elasticity (e.g. Ponte Castañeda, 2002) constitute a valuable source of inspiration.

\section{Acknowledgements}

L. Brassart and L. Delannay are mandated by the National Fund for Scientific Research (FNRS, Belgium). Computing facilities were made available through the contract FRFC-2.4502.05 with FNRS. The authors are grateful to L. Adam and N. Bilger (e-Xstream engineering SA) for providing original results obtained with the DIGIMAT-MF software.

\section{Appendix A. Derivation of the second moment of the trial strain from the effective energy of the Linear Comparison Composite}

The proof of (53) is based on the following lemma. Consider a (possibly thermoelastic) composite governed by a local convex potential $W_{t}$ depending on a parameter $t$. Then, the corresponding effective potential $\bar{W}_{t}$ also depends on t, and its derivative w.r.t. this parameter is given by (the derivative is taken with $\boldsymbol{\varepsilon}$ held fixed):

$$
\frac{\partial \bar{W}_{t}}{\partial t}(\overline{\boldsymbol{\varepsilon}})=\left\langle\frac{\partial W_{t}}{\partial t}\left(\boldsymbol{x}, \boldsymbol{\varepsilon}_{t}\right)\right\rangle
$$


where the local field $\varepsilon$ is the solution to the minimization problem:

$$
\bar{W}(t, \overline{\boldsymbol{\varepsilon}})=\inf _{\boldsymbol{\varepsilon} \in \mathcal{K}(\overline{\boldsymbol{\varepsilon}})}\left\langle W_{t}(t, \boldsymbol{\varepsilon})\right\rangle .
$$

The proof of (80) is given by Ponte Castañeda and Suquet (1998) (see also Idiart and Ponte Castañeda, 2007). It is a consequence of the chain rule, plus the fact that the effective potential $\bar{W}_{t}$ is stationary with respect to $\varepsilon_{t}$. Result (80) can be applied to the potential of the thermoelastic LCC (49), taking $t=\mu_{0}^{(r)}$ and $\bar{W}_{t}=\bar{W}_{0}$. We first obtain:

$$
\begin{aligned}
\frac{\partial W_{0}}{\partial \mu_{0}^{(r)}} & =\boldsymbol{I}^{\mathrm{dev}}::\left(\left(\boldsymbol{\varepsilon}-\boldsymbol{\varepsilon}_{n}^{p}\right) \otimes\left(\boldsymbol{\varepsilon}-\boldsymbol{\varepsilon}_{n}^{p}\right)\right) \chi^{(r)}(\boldsymbol{x}) \\
& =\frac{3}{2}\left(\boldsymbol{\varepsilon}-\boldsymbol{\varepsilon}_{n}^{p}\right)_{e q}^{2} \chi^{(r)}(\boldsymbol{x})=\frac{3}{2}\left(\varepsilon_{e q}^{t r}\right)^{2} \chi^{(r)}(\boldsymbol{x}) .
\end{aligned}
$$

Then, result (80) leads to (53).

\section{Appendix B. Numerical resolution using Newton's method}

At each time step, the homogenization procedure described in Section 5 requires the resolution of a system of $N_{p}$ equations, where $N_{p}$ is the number of phases for which the yield criterion (56) is satisfied. The residual vector is denoted by $\boldsymbol{F}$ and contains the scalar residuals $F^{(r)}(68)$ of every plastifying phase. The unknown vector is denoted by $\boldsymbol{X}$ and contains the shear moduli $\mu_{0}^{(r)}$. Accounting for Eq. (54) which relates $\Delta p^{(r)}$ to $\mu_{0}^{(r)}$, each residual $F^{(s)}$ can be viewed as a function of the macroscopic strain and of the unknown $\mu_{0}^{(r)}$. According to a Newton-Raphson scheme, the correction $\boldsymbol{c}$ applied to the current estimate of $\boldsymbol{X}$ is computed as:

$$
\boldsymbol{c}=-\boldsymbol{J}^{-1} \bullet \boldsymbol{F},
$$

where $\boldsymbol{J}$ is the sensitivity matrix, whose $\{r s\}$ component is the derivative of the $r^{\text {th }}$ component of $\boldsymbol{F}$ w.r.t. the $s^{\text {th }}$ component of $\boldsymbol{X}$, and $(\bullet)$ denotes the appropriate matrix-vector product.The components of $\boldsymbol{J}$ are given by (the notation $(\cdot)^{\prime}$ indicates a differentiation w.r.t. $\Delta p$ ):

$$
\begin{aligned}
& \left.J_{r s}=\left[-3 \mu_{0}^{(r)}+\left(\left(R^{(r)}\right)^{\prime}+\left(Z^{(r)}\right)^{\prime}\right) \frac{\mu^{(r)}-\mu_{0}^{(r)}}{\mu^{(r)}}\right)\right] \frac{\partial \sqrt{\left\langle\left(\varepsilon_{e q}^{t r}\right)^{2}\right\rangle_{r}}}{\partial \mu_{0}^{(s)}}, \quad r \neq s, \\
& \left.J_{r r}=\left[-3 \mu_{0}^{(r)}+\frac{\mu^{(r)}-\mu_{0}^{(r)}}{\mu^{(r)}}\right)\left(\left(R^{(r)}\right)^{\prime}+\left(Z^{(r)}\right)^{\prime}\right)\right] \frac{\partial \sqrt{\left\langle\left(\varepsilon_{e q}^{t r}\right)^{2}\right\rangle_{r}}}{\partial \mu_{0}^{(r)}}-\left[3+\frac{1}{\mu^{(r)}}\left(\left(R^{(r)}\right)^{\prime}+\left(Z^{(r)}\right)\right)^{\prime}\right] \sqrt{\left\langle\left(\varepsilon_{e q}^{t r}\right)^{2}\right\rangle_{r}},
\end{aligned}
$$

where the function $Z$ denotes the right-hand side member of Eq. (20). Relations (85) and (86) were obtained accounting for relation (54), which implies:

$$
\begin{aligned}
& \left.\frac{\partial \Delta p^{(r)}}{\partial \mu_{0}^{(s)}}=\frac{\mu^{(r)}-\mu_{0}^{(r)}}{\mu^{(r)}}\right) \frac{\partial \sqrt{\left\langle\left(\varepsilon_{e q}^{t r}\right)^{2}\right\rangle_{r}}}{\partial \mu_{0}^{(s)}}, \quad r \neq s, \\
& \left.\frac{\partial \Delta p^{(r)}}{\partial \mu_{0}^{(r)}}=\frac{-1}{\mu^{(r)}} \sqrt{\left\langle\left(\varepsilon_{e q}^{t r}\right)^{2}\right\rangle_{r}}+\frac{\mu^{(r)}-\mu_{0}^{(r)}}{\mu^{(r)}}\right) \frac{\partial \sqrt{\left\langle\left(\varepsilon_{e q}^{t r}\right)^{2}\right\rangle_{r}}}{\partial \mu_{0}^{(r)}} .
\end{aligned}
$$

The macroscopic consistent tangent operator is expressed by

$$
\overline{\boldsymbol{C}}^{\mathrm{alg}}=\frac{\delta \overline{\boldsymbol{\sigma}}}{\delta \overline{\boldsymbol{\varepsilon}}}=\overline{\boldsymbol{C}}_{0}+\frac{\partial \overline{\boldsymbol{\sigma}}}{\partial \boldsymbol{X}} \bullet \frac{\delta \boldsymbol{X}}{\delta \overline{\boldsymbol{\varepsilon}}},
$$

where $\delta \overline{\boldsymbol{\sigma}}$ and $\delta \boldsymbol{X}$ are variations of $\overline{\boldsymbol{\sigma}}$ and $\boldsymbol{X}$ induced by a variation $\delta \overline{\boldsymbol{\varepsilon}}$. The following results hold:

$$
\frac{\partial \overline{\boldsymbol{\sigma}}}{\partial \boldsymbol{X}}=\frac{\partial \overline{\boldsymbol{C}}_{0}}{\partial \boldsymbol{X}}: \overline{\boldsymbol{\varepsilon}}^{t r},
$$

and

$$
\frac{\delta \boldsymbol{X}}{\delta \overline{\boldsymbol{\varepsilon}}}=-\boldsymbol{J}^{-1} \bullet \frac{\partial \boldsymbol{F}}{\partial \overline{\boldsymbol{\varepsilon}}} .
$$

\section{Appendix C. Incremental (Hill-type) method for elasto-plastic composites}

Following Hill (1965a), incremental mean-field models are based on a rate formulation of the constitutive equations of the constituents:

$$
\dot{\boldsymbol{\sigma}}(\boldsymbol{x})=\boldsymbol{C}^{\operatorname{tgt}(r)}(\boldsymbol{x}): \dot{\boldsymbol{\varepsilon}}(\boldsymbol{x}), \quad \forall \boldsymbol{x} \text { in phase } r,
$$


where $\boldsymbol{C}^{\operatorname{tgt}(r)}(\boldsymbol{x})$ is the local, tangent operator. It is assumed that homogenization models for elastic composites apply to nonlinear ones considering strain rates instead of strains:

$$
\langle\dot{\boldsymbol{\varepsilon}}\rangle_{r}=\boldsymbol{A}^{(r)}: \dot{\overline{\boldsymbol{\varepsilon}}}
$$

and making use of uniform, reference tangent moduli $\widehat{\boldsymbol{C}}^{\operatorname{tgt}(r)}$ for the computation of $\boldsymbol{A}^{(r)}$, instead of elastic ones. For plastic behavior, it is well known that too stiff predictions are obtained if one uses the anisotropic tangent moduli computed for the average of the strain in each phase (Gilormini, 1995; Suquet, 1996, 1997; Chaboche et al., 2005; Pierard and Doghri, 2006b). A work-around is to consider some isotropic part of the tangent operator according to a spectral decomposition (Ponte Castañeda, 1996; Doghri and Ouaar, 2003). For J2 elasto-plasticity, the algorithmic tangent operator admits the following decomposition:

$$
\boldsymbol{C}^{\text {algo }}=3 k_{1} \boldsymbol{C}_{1}+2 k_{2} \boldsymbol{C}_{2}+2 k_{3} \boldsymbol{C}_{3},
$$

where

$$
\boldsymbol{C}_{1}=\boldsymbol{I}^{\mathrm{vol}}, \quad \boldsymbol{C}_{3}=\frac{2}{3} \boldsymbol{N} \otimes \boldsymbol{N}, \quad \boldsymbol{C}_{2}=\boldsymbol{I}^{\mathrm{dev}}-\boldsymbol{C}^{(3)},
$$

and

$$
\left.k_{1}=\kappa, \quad k_{2}=\mu \quad 1-\frac{\Delta p}{\varepsilon_{e q}^{t r}}\right), \quad k_{3}=\mu\left(1-\frac{3 \mu}{3 \mu+R^{\prime}(p)}\right) .
$$

The isotropic part of the algorithmic tangent operator according to the spectral method reads (Doghri and Ouaar, 2003):

$$
\boldsymbol{C}^{\mathrm{tgt}, \text { iso }}=3 \kappa \boldsymbol{I}^{\mathrm{vol}}+2 \mu^{\mathrm{tgt}} \boldsymbol{I}^{\mathrm{dev}}, \text { with } \mu^{\mathrm{tgt}}=k_{3} .
$$

The isotropization can be interpreted as a softening of $\boldsymbol{C}^{\text {algo }}$ in a direction orthogonal to $\boldsymbol{N}$ (Chaboche et al., 2005).

Expressions (94)-(97) are valid for incompressible plastic flow and a von Mises yield criterion. The incremental tangent approach can easily be generalized to compressible plastic flow and pressure-sensitive yield criteria, see for instance (AbouChakra Guéry et al., 2008).

\section{Appendix D. Incrementally affine method for elasto-viscoplastic composites}

As no continuum tangent operator can be derived in elasto-viscoplasticity, the rate formulation (92) of the local constitutive equations does not hold. Consequently, the incremental method described in Appendix C cannot rigorously be applied. Recently, Doghri et al. (2010) proposed an "incrementally affine" procedure which extends the incremental procedure to elasto-viscoplasticity. The formulation exploits the numerical algorithm of elasto-viscoplasticity to obtain the following discretized relation, which is given here without proof:

$$
\Delta \boldsymbol{\sigma}=\boldsymbol{C}^{\mathrm{alg}}:\left(\Delta \boldsymbol{\varepsilon}-\Delta \boldsymbol{\varepsilon}^{\mathrm{aff}}\right)
$$

where $\Delta \boldsymbol{\varepsilon}^{\text {aff }}$ is called "affine" strain increment, whose expression in $\mathrm{J}_{2}$ elasto-viscoplasticity reads:

$$
\left.\Delta \boldsymbol{\varepsilon}^{\mathrm{aff}}=\dot{p}\left(t_{n}\right) \Delta t \quad \mathbf{N}\left(t_{n}\right)+\mathbf{N}\left(t_{n+1}\right) \frac{g_{v, p}\left(t_{n+1}\right)}{1-g_{v, p} \Delta t}\right),
$$

where $g_{v}$ is the viscoplastic function, such that:

$$
\dot{p}=g_{v}(\boldsymbol{\sigma}, p)
$$

and $g_{v, p}$ its derivative w.r.t. $p$. Relation (98) defines a linear thermoelastic comparison material with stiffness $\boldsymbol{C}^{\text {alg }}$ and polarization stress $-\boldsymbol{C}^{\text {alg. }}: \Delta \boldsymbol{\varepsilon}^{\text {aff }}$. Uniform, reference moduli and polarization stresses for each phase are computed evaluating $\boldsymbol{C}^{\text {alg }}$ and $\Delta \boldsymbol{\varepsilon}^{\text {aff }}$ at the phase average of the strain. The partitioning of the strain increments among the phases is obtained using a homogenization scheme for thermoelastic composites.

Note that like for the incremental tangent scheme for rate-independent elasto-plasticity, the reference algorithmic tangent operator must be isotropized in order to obtain valid predictions. Again, the spectral method may be used (see Appendix C), leading to the following expression for $\mu^{\text {tgt: }}$

$$
\mu^{\mathrm{tgt}}=\mu\left(1-\frac{3 \mu}{3 \mu+\frac{1-g_{v, p} \Delta t}{g_{v, \sigma} \Delta t}}\right)
$$

where $g_{v, \sigma}$ is the derivative of $g_{v}$ w.r.t. $\sigma_{e q}$. 


\section{References}

ABAQUS 6.9, 2009. A General-Purpose Finite Element Software. ABAQUS Inc., Pawtucket, RI, USA

Abou-Chakra Guéry, A., Cormery, F., Shao, J., Kondo, D., 2008. A micromechanical model of elastoplastic and damage behavior of a cohesive geomaterial. Int. J. Solids Struct. 45, 1406-1429.

Benveniste, Y., 1987. A new approach to the application of Mori-Tanaka's theory in composite materials. Mech. Mater. 6, $147-157$.

Berveiller, M., Zaoui, A., 1979. An extension of the self-consistent scheme to plastically-flowing polycrystals. J. Mech. Phys. Solids 26 , 325-344.

Bobeth, M., Diener, G., 1986. Field fluctuations in multicomponent mixtures. J. Mech. Phys. Solids 34, 1-17.

Brassart, L., 2011. Homogenization of Elasto-(Visco)Plastic Composites: History-Dependent Incremental and Variational Approaches. Ph.D. Thesis. Ecole Polytechnique de Louvain, Université catholique de Louvain. <http://dial.academielouvain.be/handle/boreal:75974>.

Brassart, L., Doghri, I., Delannay, L., 2010. Homogenization of elasto-plastic composites coupled with a nonlinear finite element analysis of the equivalent inclusion problem. Int. J. Solids Struct. 47, 716-729.

Brassart, L., Stainier, L., 2012. On convergence properties of variational constitutive updates for elasto-visco-plasticity. GAMM Mitt. 35, 26-42.

Brassart, L., Stainier, L., Doghri, I., Delannay, L., 2011. A variational formulation for the incremental homogenization of elasto-plastic composites. J. Mech. Phys. Solids 59, 2455-2475.

Brenner, R., Castelnau, O., Gilormini, P., 2001. A modified affine theory for the overall properties of nonlinear composites. C. R. Acad. Sci. Paris Sér. II b, 649654.

Canadija, M., Mosler, J., 2011. On the thermomechanical coupling in finite strain plasticity theory with non-linear kinematic hardening by means of incremental energy minimization. Int. J. Solids Struct. 48, 1120-1129.

Carstensen, C., Hackl, K., Mielke, A., 2002. Non-convex potentials and microstructures in finite-strain plasticity. Proc. R. Soc. Lond. A $458,299-317$.

Chaboche, J.-L., 2008. A review of some plasticity and viscoplasticity constitutive theories. Int. J. Plasticity 24, 1642-1693.

Chaboche, J.-L., Kanouté, P., 2003. Sur les approximations “isotrope" et "anisotrope" de l'opérateur tangent pour les méthodes tangentes incrémentale et affine. C. R. Mécanique 331, 857-864.

Chaboche, J.L., Kanouté, P., Roos, A., 2005. On the capabilities of mean-field approaches for the description of plasticity in metal matrix composites. Int. J. Plasticity 21, 1409-1434.

Chaboche, J.-L., Kruch, S., Maire, J., Pottier, T., 2001. Towards a micromechanics based inelastic and damage modeling of composites. Int. J. Plasticity 17, 411439.

Comi, C., Corigliano, A., Maier, G., 1991. Extremum properties of finite-step solutions in elastoplasticity with nonlinear hardening. Int. J. Solids Struct. 27, 965-981.

DIGIMAT, 2011. Software Platform for Nonlinear Multi-scale Modeling of Composite Materials and Structures. e-Xstream Engineering, Belgium and Luxembourg. <www.e-Xstream.com>.

Doghri, I., 2000. Mechanics of Deformable Solids: Linear and Nonlinear, Analytical and Computational Aspects. Springer.

Doghri, I., Adam, L., Bilger, N., 2010. Mean-field homogenization of elasto-viscoplastic composites based on a general incrementally affine linearization method. Int. J. Plasticity 26, 219-238.

Doghri, I., Brassart, L., Adam, L., Gérard, J.-S., 2011. A second-moment incremental formulation for the mean-field homogenization of elasto-plastic composites. Int. J. Plasticity 27, 352-371.

Doghri, I., Friebel, C., 2005. Effective elasto-plastic properties of inclusion-reinforced composites. study of shape, orientation and cyclic response. Mech. Mater. 37, 45-68.

Doghri, I., Ouaar, A., 2003. Homogenization of two-phase elasto-plastic composite materials and structures: study of tangent operators, cyclic plasticity and numerical algorithms. Int. J. Solids Struct. 40, 1681-1712.

Dvorak, G., 1992. Transformation Field Analysis of inelastic composite materials. Proc. Roy. Soc. Lond. A 437, 311-327.

Dvorak, G., Benveniste, Y., 1992. On transformation strains and uniform fields in multiphase elastic media. Proc. R. Soc. Lond. A 437, 291-310.

Eshelby, J.D., 1957. The determination of the elastic field of an ellipsoidal inclusion, and related problems. Proc. R. Soc. Lond. A 241, 376-396.

Franciosi, P., Berbenni, S., 2008. Multi-laminate plastic-strain organization for non-uniform TFA modeling of poly-crystal regularized plastic flow. Int. J. Plasticity 24, 1549-1580.

Fritzen, F., Böhlke, T., 2010. Three-dimensional finite element implementation of the nonuniform transformation field analysis. Int. J. Numer. Methods Eng. $84,803-829$.

Germain, P., Nguyen, Q., Suquet, P., 1983. Continuum thermodynamics. J. Appl. Mech. 50, 1010-1020.

Gilormini, P., 1995. Insuffisance de l'extension classique du modèle auto-cohérent au comportement non linéaire. C. R. Acad. Sci. Paris 320 (Série IIb), 115122.

González, C., LLorca, J., 2000. A self-consistent approach to the elasto-plastic behaviour of two-phase materials including damage. J. Mech. Phys. Solids 48, 675-692.

González, C., Segurado, J., LLorca, J., 2004. Numerical simulation of elasto-plastic deformation of composites: evolution of stress microfields and implications for homogenization models. J. Mech. Phys. Solids 52, 1573-1593.

Halphen, B., Nguyen, Q., 1975. Sur les matériaux standards généralisés. J. Mécanique 14, 39-63.

Hashin, Z., 1970. Complex moduli of viscoelastic composites - I. General theory and application to particulate composites. Int. J. Solids Struct. 6, 539-552.

Hill, R., 1965a. Continuum micro-mechanics of elastoplastic polycrystals. J. Mech. Phys. Solids 13, 89-101.

Hill, R., 1965b. A self-consistent mechanics of composite materials. J. Mech. Phys. Solids 13, 213-222.

Idiart, M., Ponte Castañeda, P., 2007. Field statistics in nonlinear composites. I. Theory. Proc. R. Soc. Lond. A 463, $183-202$.

Kreher, W.S., 1990. Residual stresses and stored elastic energy of composites and polycrystals. J. Mech. Phys. Solids 38, 115-128.

Kröner, E., 1958. Berechnung der elastischen konstanten des vielkristalls aus den konstanten des einkristalls. Z. Phys. 151, $504-518$.

Lahellec, N., Suquet, P., 2007a. On the effective behavior of nonlinear inelastic composites: I. Incremental variational principles. J. Mech. Phys. Solids 55, 1932-1963.

Lahellec, N., Suquet, P., 2007b. On the effective behavior of nonlinear inelastic composites: II. A second-order procedure. J. Mech. Phys. Solids 55, $1964-1992$.

Lebensohn, R., Tomé, C., 1993. A self-consistent anisotropic approach for the simulation of plastic deformation and texture development of polycrystals: application to zirconium alloys. Acta Metall. Mater. 41, 2611-2624.

Lemaître, J., Chaboche, J.-L., 1990. Mechanics of Solid Materials. Cambridge University Press.

Li, J., Weng, G., 1997. A secant-viscosity approach to the time-dependent creep of an elastic-viscoplastic composite. J. Mech. Phys. Solids 45, $1069-1083$.

Li, J., Weng, G., 1998. A unified approach from elasticity to viscoelasticity to viscoplasticity of particle-reinforced solids. Int. J. Plasticity 14 , 193-208.

Masson, R., Bornert, M., Suquet, P., Zaoui, A., 2000. An affine formulation for the prediction of the effective properties of nonlinear composites and polycrystals. J. Mech. Phys. Solids 48, 1203-1227.

Masson, R., Zaoui, A., 1999. Self-consistent estimates for the rate-dependent elastoplastic behaviour of polycrystalline materials. J. Mech. Phys. Solids 47, $1543-1568$.

Maugin, G.A., 1992. The Thermomechanics of Plasticity and Fracture. Cambridge University Press.

Michel, J., Suquet, P., 2003. Nonuniform transformation field analysis. Int. J. Solids Struct. 40, 6937-6955.

Michel, J., Suquet, P., 2004. Computational analysis of nonlinear composite structures using the non-uniform transformation field analysis. Comput. Methods Appl. Mech. Eng. 193, 5477-5502.

Miehe, C., 2002. Strain-driven homogenization of inelastic microstructures and composites based on an incremental variational formulation. Int. J. Numer. Methods Eng. 55, 1285-1322. 
Molinari, A., Canova, G.R., Azhi, S., 1987. A self-consistent approach to the large deformation polycrystal viscoplasticity. Acta Metall. Mater. 35, $2983-2994$. Mori, T., Tanaka, K., 1973. Average stress in matrix and average elastic energy of materials with misfitting inclusions. Acta Metall. 21, $571-574$.

Mosler, J., 2010. On variational updates for non-associative kinematic hardening of Armstrong-Frederick-type. Tech. Mech. 30, $244-251$.

Nyssen, C., 1981. An efficient and accurate iterative method, allowing large incremental steps, to solve elasto-plastic problems. Comput. Struct. $13,63-71$. Ortiz, M., Stainier, L., 1999. The variational formulation of viscoplastic constitutive updates. Comput. Methods Appl. Mech. Eng. 171, 419-444.

Perzyna, P., 1964. On the constitutive equations for work-hardening and rate sensitive plastic materials. Bull. Acad. Polon. Sci. Sér. Sci. Technol. 12, 199-206.

Pierard, O., Doghri, I., 2006a. An enhanced affine formulation and the corresponding numerical algorithms for the mean-field homogenization of elastoviscoplastic composites. Int. J. Plasticity 22, 131-157.

Pierard, O., Doghri, I., 2006b. Study of various estimates of the macroscopic tangent operator in the incremental homogenization of elastoplastic composites. Int. J. Multiscale Comput. Eng. 4, 521-543.

Pierard, O., Gonzalez, C., Segurado, J., LLorca, J., Doghri, I., 2007a. Micromechanics of elasto-plastic materials reinforced with ellipsoidal inclusions. Int. J. Solids Struct. 44, 6945-6962.

Pierard, O., LLorca, J., Segurado, J., Doghri, I., 2007b. Micromechanics of particle-reinforced elasto-viscoplastic composites: finite element simulations versus affine homogenization. Int. J. Plasticity 23, 1041-1060.

Ponte Castañeda, P., 1991. The effective mechanical properties of nonlinear isotropic composites. J. Mech. Phys. Solids 39, 45-71.

Ponte Castañeda, P., 1996. Exact second-order estimates for the effective mechanical properties of nonlinear composite materials. J. Mech. Phys. Solids 44, $827-862$.

Ponte Castañeda, P., 2002. Second-order homogenization estimates for nonlinear composites incorporating field fluctuations: I - theory. J. Mech. Phys. Solids 50, 737-757.

Ponte Castañeda, P., Suquet, P., 1998. Nonlinear composites. Adv. Appl. Mech. 34, 171-302.

Rekik, A., Auslender, F., Bornert, M., Zaoui, A., 2007. Objective evaluation of linearization procedures in nonlinear homogenization: A methodology and some implications on the accuracy of micromechanical schemes. Int. J. Solids Struct. 44, 3468-3496.

Segurado, J., LLorca, J., González, C., 2002. On the accuracy of mean-field approaches to simulate the plastic deformation of composites. Scripta Mater. 46, 525-529.

Simo, J.C., Hughes, T., 1998. Computational Inelasticity. Springer.

Suquet, P., 1995. Overall properties of nonlinear composites: a modified secant moduli theory and its link with Ponte Castañeda's nonlinear variational procedure. C.R. Acad. Sci. Paris Sér. IIb 320, 563-571.

Suquet, P., 1996. Overall properties of nonlinear composites: remarks on secant and incremental formulations. In: Zaoui, A., Pineau, A. (Eds.), Micromechanics of Plasticity and Damage of Multiphase Materials. Kluwer Academic Publishers, Dordrecht, pp. 149-156.

Suquet, P., 1997. Effective properties of nonlinear composites. In: Suquet, P. (Ed.), Continuum Micromechanics, CISM Lecture Notes, vol. 377. Springer Verlag, New York, pp. 197-264.

Tandon, G.P., Weng, G.J., 1988. A theory of particle-reinforced plasticity. J. Appl. Mech. 55, 126-135.

Weinberg, K., Mota, A., Ortiz, M., 2006. A variational constitutive model for porous metal plasticity. Comput. Mech. 37, 142-152.

Wilkins, M., 1964. Calculation of elasto-plastic flow. In: Alder, B. et al. (Eds.), Methods of Computational Physics, vol. 3. Academic Press, New York.

Zaoui, A., 2002. Continuum micromechanics: survey. J. Eng. Mech. 128, 808-816. 